\title{
Active integration of electric vehicles into distribution grids: barriers and frameworks for flexibility services
}

\author{
Felipe Gonzalez Venegas ${ }^{\mathrm{a}, \mathrm{b}, *}$, Marc Petit $^{\mathrm{a}}$, Yannick Perez ${ }^{\mathrm{c}}$ \\ ${ }^{a}$ Université Paris-Saclay, CentraleSupélec, CNRS, Laboratoire de Génie Electrique et Electronique de \\ Paris, 91192, Gif-sur-Yvette, France. \\ ${ }^{b}$ Innovation 83 Advanced Technologies Research Division, Groupe PSA, 78955, Carrières-sous-Poissy, \\ France \\ ${ }^{c}$ Université Paris-Saclay, CentraleSupélec, Laboratoire de Génie Industriel 91190, Gif-sur-Yvette, France.
}

\begin{abstract}
Distribution system operators face a challenging environment marked by increased decentralization, digitalization, and the decarbonization of transport and heating sectors. In particular, the integration of large numbers of electric vehicles (EVs) will pose challenges for distribution grid operation and planning. However, EVs also open the opportunity to offer flexibility services to different actors in the electricity system using smart charging and vehicle-to-grid (V2G) technology. This work reviewed the scientific literature and key European demonstrator projects on the proactive integration of EVs into distribution grids. The main technical, economic, regulatory, and user-related aspects were analyzed and the associated barriers identified. There is a broad scientific literature on the technical feasibility of EV flexibility provision and coordination schemes, which has as well been proved in demonstrator projects, even though the required technologies for $\mathrm{V} 2 \mathrm{G}$ (bidirectional chargers, communication protocols) are not yet widespread. On the other hand, main barriers are economic and institutional, largely due to a lack of regulatory frameworks to value flexibility at distribution level and thus uncertainty on the value of these flexibility services. In particular, this work analyzed four possible value frameworks (grid codes, connection agreements, tariffs and market platforms) to use flexibility at the distribution level, and their implementations with EV fleets in demonstrator projects.
\end{abstract}

\footnotetext{
${ }^{*}$ Corresponding author

Email address: felipe.gonzalezvenegas@centralesupelec.fr. Address: CentraleSupelec, 3 rue Joliot Curie, 91190, Gif-sur-Yvette France. (Felipe Gonzalez Venegas)
} 


\section{Highlights}

- Holistic review of scientific literature and EV demonstrator projects.

- Identification of flexibility services that EVs can provide throughout the value chain.

- Identification of technical, economic and regulatory barriers for active EV integration.

- Analysis of possible frameworks to use EV-driven flexibility at distribution level.

Word Count: 9999

Keywords: Electric vehicles, Electricity distribution systems, Smart grids, Flexibility Markets, Vehicle-to-grid, Smart Charging

\section{Introduction}

Environmental challenges are pushing the electrification of the transport sector. Electric vehicles (EV), if coupled with a low-carbon electricity generation matrix, can greatly reduce $\mathrm{CO}_{2}$ emissions of the transport sector while also cutting the emissions of other atmospheric pollutants, such as particulate matter and $\mathrm{NO}_{\mathrm{x}}$, and noise levels [1]. These reasons have prompted many governments and cities to adopt pro-EV measures [2], such as stricter $\mathrm{CO}_{2}$ emissions standards and low emission zones, that push car manufacturers to improve fuel efficiency of combustion engines and develop new electric models. In Europe, car manufacturers need to respect an average fleet emission of $95 \mathrm{gCO}_{2} / \mathrm{km}$ since $2020 / 21$, which represents, on average, a 21\% reduction from 2018 emission values [3]. These factors have spurred rapid growth of the EV market, with over 3.2 million EVs sold in 2020, an increase of $43 \%$ from 2019 [4]. This trend is expected to continue, reaching over 200 millions EVs in the streets by 2030, according to IEA's favorable scenario [2].

On the other hand, distribution system operators (DSO) face significant challenges related to the integration of distributed renewable energy sources (RES), such as wind farms and PV panels, novel schemes to empower prosumers, such as energy communities, and cross-sector electrification, such as heating and transport [5. However, information and communication technologies (ICTs) are enabling the control and monitoring of distributed energy resources 


\begin{tabular}{ll} 
Abbreviations & \\
\hline EV & Electric vehicle \\
DSO & Distribution system operator \\
TSO & Transmission system operator \\
BRP & Balancing responsible party \\
OEM & Original equipment (automobile) manufacturers \\
RES & Renewable energy sources \\
DER & Distributed energy resources \\
PV & Solar photovoltaic system \\
LV/MV/HV & Low/medium/high voltage \\
NWA & Non-wire alternative \\
V2X/G/H/B & Vehicle-to-grid/home/building (bidirectional charging) \\
VGI & Vehicle-Grid integration \\
SoC & State-of-Charge \\
ICT & Information and communication technologies \\
PCI & Public charging infrastructure \\
EVSE & Electric vehicle supply equipment (charging station) \\
LEM & Local energy market \\
CEER &
\end{tabular}


(DER), including EVs and RES, which can provide flexibility for improved operation and planning of the power grids.

In particular, EV integration is set to impact distribution networks. These impacts can be categorized as load and voltage issues. Additional EV load can increase active power losses and create congestion in distribution grid assets. Overloading of transformers or lines can cause equipment degradation and failure. Voltage issues affect the quality of service delivered to end-users, which should be maintained in a suitable range (EN50160 standard in Europe [6]). EV charging can create voltage drops and phase-unbalances beyond these grid requirements. These impacts will depend on several factors including EV penetration rate, grid topology, user behavior and tariff schemes [7]. To deal with these issues, DSOs would need to invest in infrastructure reinforcement, upgrading transformers or lines to alleviate congestion or keep the voltage within the required boundaries. Note that these same constraints can arise due to the integration of other DERs as well, such as distributed generation or electrical heating devices [8].

Even though EVs carry impacts for power systems, they also carry great flexibility potential. In this context, flexibility is defined as a controlled power adjustment sustained for a required duration [9]. EVs are idle over $80 \%$ of the time [10] and their average daily consumption can be charged in under 3 hours with a standard $3.7 \mathrm{kVA}$ home charger [11]. This leaves ample margin for controlling the charging process (smart charging) and even using the EV as a storage system that can give power back to the home (V2H), building (V2B) or the grid (V2G). In the case of distribution grids, EV flexibility can be used to defer or avoid costly infrastructure reinforcements, with great economic savings. My Electric Avenue project estimated that by 2050 a third of low voltage (LV) grids in the UK would need reinforcements (with an EV uptake at 40-70\%), but a simple coordination system could generate up to $£ 2.2$ billion in investment savings [12].

To fully make use EV flexibility, a series of technical and economic factors must first be addressed. In particular, a framework for the procurement of flexibility by DSOs has to be created. This type of framework exists for the procurement of flexibility services by transmission system operators (TSO) in the form of balancing markets. The rules governing these markets are evolving to allow DERs to participate as service providers [13]. However, 
at distribution level, the mechanisms to procure flexibility for the operation and planning of the grid are still under development.

This works seeks to analyze the key aspects to address for proactive integration of EVs into distribution systems by providing flexibility for grid operation and planning. The main contribution of this work is the identification of the associated main technical, economic and regulatory barriers, as well as emerging opportunities that mobilize the latest developments in exploitation of flexibility at the local level.

This paper starts by presenting the methodological analysis framework, followed by a detailed analysis of its technical, economic, regulatory and social components. A discussion summarizing the key barriers to exploiting EV flexibility at the local level and potential leads for future research arising from recent regulatory developments in Europe concludes the paper.

\section{Methodological analysis framework}

EVs will integrate the smart grid ecosystem, where they will interact with other emerging technologies, such RES and stationary batteries (BESS), as well as with various stakeholders, such as aggregators (an agent that controls a pool of flexibility resources, such as EVs, to participate in electricity markets or to offer flexibility to other market players) and system operators (DSOs, TSOs). The complex interactions between all these agents depend on many factors, including technological developments, economic relationships, regulatory frameworks and EV-user preferences and behavior. To properly analyze these interactions, a methodological framework, proposed in [14] for PV-EV synergies, was adapted and applied to the EV-distribution grid relationship.

By reviewing the scientific literature and the results and recommendations of main European demonstrator projects concerning smart grids and electric vehicle grid integration (VGI), key factors to address in order to exploit EV flexibility were identified, as shown in Figure 1. These aspects can be divided in technical, economic, regulatory and user-related aspects.

Technical aspects consist on the strategies adopted to use EV flexibility, and how they are implemented. These strategies are applied in a given spatial configuration of the electrical 


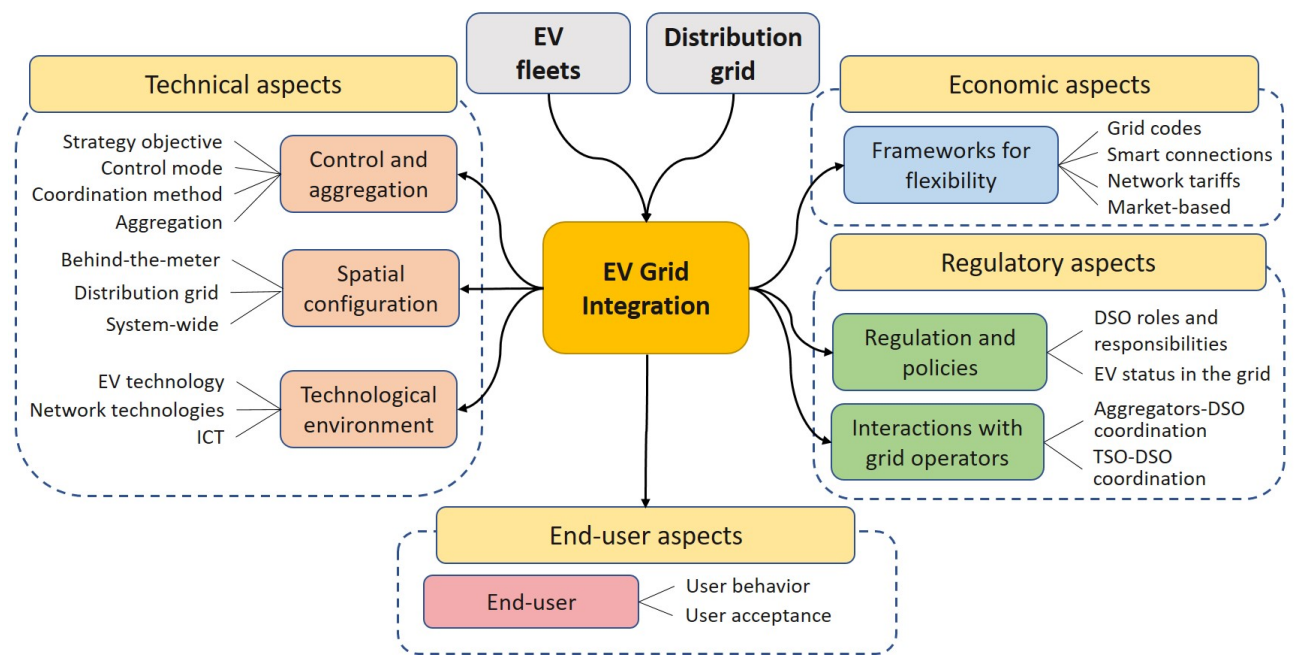

Figure 1: Analytical framework, adapted from [14]

grid, from user-centered (behind-the-meter), to the local network (LV and MV distribution grids) or system-wide level (transmission level, wholesale markets). Additionally, VGI will be determined by the technological environment in which it develops, which encompasses the EV charging technology, the interaction with other DERs, and governing ICT standards and requirements.

Understanding economic and regulatory aspects is crucial for successful EV integration and robust business models for flexibility services. The main aspects are related to evolving policy and regulation to allow and encourage flexibility trading at distribution level, not only for EVs but for other types of demand response mechanisms as well, to how innovative frameworks for flexibility procurement can be implemented at the distribution level and to interactions between stakeholders.

Finally, the implementation of any flexibility service will depend on end-user behavior (how EVs are used and charged) and acceptance of the control strategy. While these aspects are often overlooked or misrepresented in academic studies, they are key to the success of its deployment [15]. 


\section{Technical aspects}

\subsection{Control and aggregation}

Smart control is the main lever to exploit EV flexibility and it refers to the technical implementation of EV charging strategies for flexibility provision. Key characteristics are the strategy objective, the control mode, the coordination method and aggregation issues.

\subsubsection{Strategy objective}

The strategy objective is the core of smart control. It sets the management of the EV charging process to respond to the user or another agent needs. The objective can represent a monetary or a physical quantity and may be directly tied to the flexibility services potentially offered to the agents. There are many proposed strategy objectives that may interest various stakeholders [16]:

- For end-users: Optimizing EV charging costs and management of electricity bills and assets (by means of V2H/V2B). This can consider increasing self-consumption of local renewable generation and optimizing the contracted power.

- For DSOs: Management of grid assets (congestion and voltage support), power losses minimization, valley filling and phase balancing (in LV grids).

- For TSOs or Balancing Responsible Parties (BRPs): Provision of balancing services (including frequency regulation), optimization of system-wide generation costs and RES support.

Several objectives can be targeted jointly, such as optimizing end-user costs while safeguarding the limits of the distribution grid operation, thus needing a hierarchy or common value system to discriminate among them. For an in-depth review of algorithms and objectives for smart charging and V2G, refer to [17, 18, 19, 20, 21].

\subsubsection{Control mode}

Control mode refers how the strategy objective is implemented in the EV environment. It sets a strategy objective with diverse constraints coming from end-users (charging requirements, EV technology), distribution grids (capacity limits, voltages standards) or other technologies (RES generation), all in a mathematical model. Control mode can be set through 
optimization, heuristics or hybrid techniques, and is applied in different time frames, either scheduling (day-ahead or intra-day) or in real-time.

Optimization techniques seek to minimize (or maximize) an objective function, representing the strategy objective of EV charging, subjected to a set of constraints. For example a charging strategy could search to minimize charging costs, constrained to a certain minimum state-of-charge (SoC) of the battery at the end of the charging process, or to respect the operating limits of the grid, such as current and voltage limits. Optimization techniques are usually used for day-ahead or intra-day scheduling of EV charging. According to the model employed they require forecast data, namely EV energy requirements, arrival and departure times, electricity prices, load profiles, RES generation, among others [22]. Most models are deterministic, assuming accurate forecasts. To represent uncertainty, stochastic optimization can be used by modeling several scenarios coming from different forecasts, though this technique is computationally more demanding [23]. Real-time optimization can be implemented to account for forecast errors, but it can be computational burdensome for large-scale fleets 24

Heuristic techniques use a set of rules or algorithms to determine the charging process. These type of algorithms are usually used in real-time control, as they are easier to implement and often require less data or communication than optimization methods. Heuristics techniques come in many forms, for example, [25] proposed a rule-based algorithm for thermal management of a neighborhood MV/LV transformer, [26] proposed a droop-based controller for real-time voltage and local congestion management using only local measurements and [27] proposed an algorithm based on game-theory to jointly reduce transformer overloading, grid losses and charging costs for the end-user.

Finally, hybrid techniques combine both optimization and heuristic approaches, usually in a two-stage process. In [28], the authors implemented a two-stage hierarchical control strategy, where the upper stage runs local congestion management using optimization over an aggregated EV fleet, and the lower stage implements decentralized voltage support using heuristics. Similarly, [29] proposed a day-ahead optimal fleet management to minimize network charges for DSOs, followed by a real-time fuzzy-logic control method that tries to follow the programmed schedule. 


\subsubsection{Coordination method}

Smart control strategies often require the coordination of large EV fleets, which can be done in a centralized or decentralized manner. In centralized control, the entire fleet is commanded by a single central entity. This coordination method has the advantage of leading to globally optimal solutions, thus supporting higher EV penetration rates, but at a cost of higher communication, infrastructure, and data transfers requirements, as well as increasing computational requirements when the aggregated number of EVs increases. In decentralized control, charging decisions are taken by each EV, based on local measures or with limited information exchange with a central aggregator. This method can arrive at similar outcomes (near-optimal) to centralized control, especially at low EV penetration rates, and has lower ICT requirements. However, it may not provide optimal responses with higher EV penetration or under forecast errors [30] [27].

\subsubsection{Aggregation}

The control strategy can be implemented by an aggregator who will act as the intermediary between EVs and electricity markets or system operators. The aggregator will be responsible for gathering the flexibility of distributed resources, offering it to markets/market agents, and then controlling the pool of resources accordingly (i.e., implementing the strategy, control, and coordination methods previously discussed).

Aggregators need to assess and propose a flexibility offer to market agents which transmits the ability to provide flexibility in a concise manner [31] [24]. A flexibility offer is usually comprised by an expected load profile and a space of possible deviations from it. This requires advanced forecasting and modeling of the flexibility resources, which in the case of EV fleets means knowledge of the users' driving and charging behavior and modeling of technical constraints. Flexibility aggregation allows other market agents (DSOs, TSOs) to optimize their operations without facing all the technical constraints from the distributed resources.

\subsection{Spatial configuration}

The control strategy is applied within a given spatial configuration: behind-the-meter, distribution grid or system-wide. At each level, flexibility will be used to meet the requirements of different stakeholders and will require control over different EV fleet sizes. 


\subsubsection{Behind-the-meter}

Behind-the-meter strategies consist on applications for end-users, behind the point of connection to the distribution grid. Applications have been proposed for homes (V2H), residential and work buildings (V2B) [32] [33], parking lots, and more [34]. EV flexibility can be used for bill optimization based on electricity tariffs, for load management based on maximum connection capacity, for increasing self-consumption of renewable energy (mainly from rooftop PV), and also as a backup power system (islanding capacity) [14] 35]. These strategies are designed for households, controlling one or two EVs, and for residential buildings or commercial/industrial facilities, controlling small EV fleets.

\subsubsection{Distribution grid}

Distribution grids supply electricity to end-customers and serve as point of connection for industry and small/medium-scale distributed generation facilities, like wind and solar farms. They distribute electricity from the transmission system to end-users via MV (2 to $36 \mathrm{kV}$ ) and LV grids (230V phase-to-ground in Europe, $110 \mathrm{~V}$ in the US) [36].1

EV flexibility can serve for local congestion management and voltage regulation in MV and LV grids. Phase balancing in LV grids (due to single-phase connections of end-users) as well as valley-filling or peak shaving services, can serve to improve quality of service and reduce active losses in the grid [37].

Flexibility at the distribution level can be used at various timeframes. At the mediumto long-term, flexible assets can defer or avoid grid reinforcements (such as transformers and feeders) by reducing congestion or voltage issues. At the operational time-frame, flexibility assets can improve reliability of the grid during congestion periods, for example due to seasonal peak load or high local renewable generation, during scheduled maintenance, which can temporarily reduce network capacity, and during post-fault (restoration) events [38] 39] [40].

Applications at distribution level would need to manage dozens of EVs in LV grids, equivalent to tens to hundreds of $\mathrm{kW}$ (neighborhood scale in Europe), up to hundreds or even thousands of EVs in MV grids, equivalent to several MW (mid-size urban neighborhood or large rural areas).

\footnotetext{
${ }^{1}$ In some countries distribution can also include the high voltage (HV) grid up to $110 \mathrm{kV}$ [36]
} 


\subsubsection{System-wide level}

At system-wide level, the main concerns are efficient and secure operation of the regional or national power system. For this, electricity markets are organized at various timeframes. At longer timeframes, capacity markets ensure sufficient generation is available to cope with peak demand. On operational time-frames (day-ahead and intraday) energy is traded among market participants. Finally, to ensure secure and reliable operation, TSOs implement balancing markets to procure reserves that can be activated in real-time if unbalances are detected between demand and supply.

EV flexibility can serve to provide system-wide services such as frequency containment reserves and energy arbitrage for BRPs, and can serve the capacity market as demand side response. Provision of balancing reserves using EV fleets has been done commercially in the PJM interconnection (US) since 2013 [41] and in demonstrator projects such as Parker in Denmark [42] or GridMotion in France [43]. Energy arbitrage consists in adapting the charging process according to BRPs' strategies in the electricity markets, thus capturing value from price differences in the electricity market [44]. This can provide benefits for operation of the electricity system, reducing generation costs [45] and supporting the integration of renewable energy [46].

However, if not managed correctly, the activation of flexibility for system-wide purposes can create congestion at the distribution level. For example, EVs providing frequency response services to the TSO [47] or reacting to low electricity prices due to high renewable generation periods can increase congestion in the distribution grid due to synchronized charging of EVs [48].

Facilitating renewable integration by means of smart charging and V2G is a topic relevant to all levels of the grid. For end-users, EV-PV complementarities can help them reduce their energy bills, and self-consumption at the residential or district level can improve renewable grid integration [49]. The coordination of EV charging and local renewable generation can reduce voltage issues and congestion of grid assets at the distribution level, and at the transmission level it can improve the operation of the generation fleet, increasing RES hosting capacity and/or reducing RES curtailment [50].

Figure 2 summarizes the different flexibility services to be provided by EVs. 


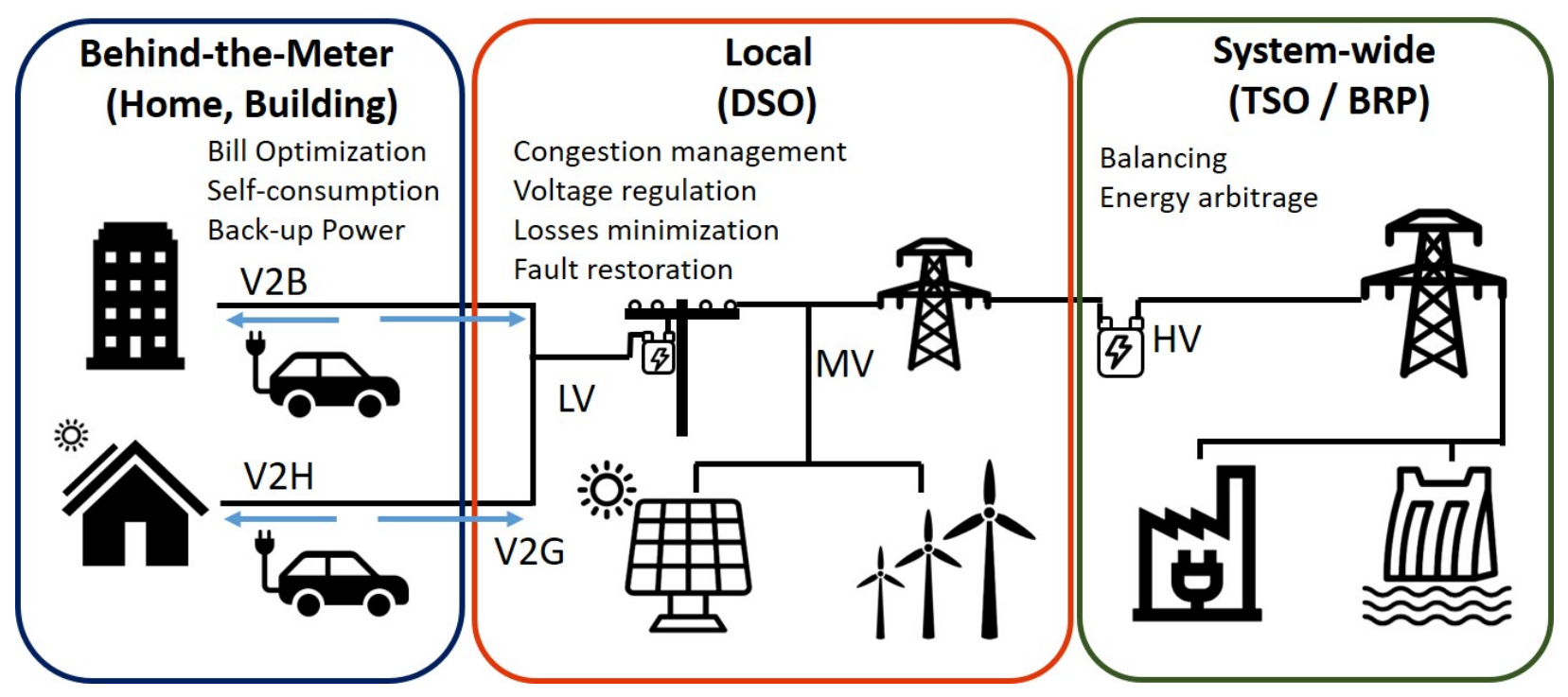

Figure 2: Main flexibility services to be provided by EVs

\subsection{Technological Environment}

EVs will integrate a rapidly changing environment as new technologies break through. The smart grid paradigm is changing the operation of distribution networks, opening up the possibility of synergies between EVs and other technologies. Core factors in the technology environment can be classified into EV charging technologies, network technologies and ICTs. OEMs need to know the technical requirements regarding charging equipment and ICTs that have to be developed and deployed in future EVs to make them smart grid compliant.

\subsubsection{EV charging technology}

Charging technology and its deployment have a direct effect on the impacts of EVs in power systems and in the flexibility services that they can provide.

$\mathrm{An} \mathrm{AC} / \mathrm{DC}$ converter system is required to charge the battery, which can be implemented on-board (inside the EV) or off-board (in the charging station, EVSE). To implement advanced smart charging and V2G, a dedicated EVSE is required for control and protection functions (mode 3 for AC or mode 4 for DC charging, according to IEC 61851-1 standard [51]).

Charging technology characteristics and capabilities will affect EV integration. EVs are large loads: a single-phase home charging station (3.7 to $7.4 \mathrm{kVA})$ is in the range of a typical household and can have significant impacts in LV grids. Increasing charging power (for 
example from 3.7 to $7.4 \mathrm{kVA}$ ) can create greater stress in the distribution grid [52], but it can also increase the flexibility potential of EV fleets. For example, moving from $3 \mathrm{kVA}$ chargers to 7 or $11 \mathrm{kVA}$ ones can increase frequency response provision by EV fleets [25] [44]. There are other technical characteristics that can affect the flexibility potential of EVs, such as accuracy to control signals, response times and efficiency of the charging equipment. The efficiency of charging equipment is extremely important, as high energy losses during charging and discharging processes may hinder the economic viability of V2G-based flexibility services [53].

Capabilities of charging equipment will also determine the flexibility services that EVs will be able to provide. Proposed smart control strategies take advantage of various capabilities that are not yet universally deployed in EVs and charging infrastructures, such as bidirectional flows and reactive power provision. Bidirectional chargers are needed to exploit V2X and use EVs as distributed storage units, but currently there are few bidirectional chargers and compatible EVs available in the market. Technical challenges (in particular improving round-trip efficiency) and high costs still need to be overcome [15]. Controllable reactive power provision has been proposed for voltage regulation at LV and MV grids [54] [55], but today's chargers do not provide this capability. This may change with ongoing R\&D by OEMs, since this feature could be required by grid codes for bidirectional chargers. Renault, for example, tested a grid-code compliant on-board V2G charger [56].

Finally, battery degradation may represent a major impediment to V2G-based services, as V2G-induced additional battery cycling can reduce the battery's lifespan. Battery aging may significantly impact the viability of business models for flexibility services, and is a major factor in end-user acceptance of V2G [57]. Battery degradation is a complex process, ruled principally by two behaviors: calendar aging, dependent on temperature and SoC at storage, and cycling aging, dependent on power throughput, depth of discharge and other factors [58]. Recent studies have shown that V2G might significantly reduce battery life if not used properly [59], but have only minor effects if its usage is limited (20 times a year for energy-intensive services like peak-shaving, or for low-impact frequency response) [60]. If peak shaving services are used daily, they can have significant impact on battery degradation 61]. 
Algorithms and best practices have been proposed to enhance battery life with smart charging and V2G, but further studies are needed for accurate battery characterization and experimental validation, as they are chemical composition dependent and most results come from simulations [58] 62 .

\subsubsection{Smart grid technologies}

EVs will interact with other technologies in future smart grids, where different flexibility resources will actively help improving the operation of distribution grids. The main technologies are RES, other flexibility sources, such as BESS and demand response, and network technologies that allow improved operation and control of grid topology (such as soft open points [63], LV on-load tap changers and other power electronics equipment [64]).

The interaction of EVs and distributed generation has been widely studied at different levels. By using renewable energy to charge the EVs, synergies may appear, allowing increased penetration rates of both technologies, and reducing curtailed renewable energy [65] 66] 67] [50]. V2G-capable EVs can also increase the integration of other technologies, such as heat pumps in residential neighborhoods [68].

Other flexibility technologies such as BESS and demand response mechanisms can support EV integration, acting as complementary flexibility sources. Aggregators can group different assets together, such as EVs, BESS and demand response, to participate in local or wholesale energy and flexibility markets. This would enable aggregators to reduce risk, by relying on other resources when EVs are unavailable. Second-life EV batteries can be used for distribution grid support services, thus allowing increased EV penetration and fostering a flexibility asset ecosystem throughout the EV battery value-chain [69] [70].

\subsubsection{Information and communication technologies}

Future smart grids need advanced ICT systems. The development of grid services requires advanced metering, control, and transactional communication involving several agents: EVs, aggregators, DSOs, TSOs, market operators, and other market players. Communication protocols and infrastructure need to be defined and deployed for the provision of flexibility services [22][71].

First, DSOs cannot develop flexibility services without greater visibility of their grids, 
i.e. grid status monitoring at near-real time resolution. Greater grid visibility will provide a better knowledge of grid utilization and flexibility services requirements. Smart meters are being deployed in many parts of the world, but with a variety of technical characteristics that may not be suitable for flexibility services settlement. In particular, smart meters frequency sampling should be aligned with flexibility trading periods and serve as settlement meters in order to avoid extra costs for participation in flexibility mechanisms [22]. Recent projects have found that requirements on settlement meters for DERs may undermine the economic viability of these solutions [72][15].

Second, communication protocols are required between different infrastructures and stakeholders: EVs, EVSE, flexibility aggregators, DSO/TSO, and commercial stakeholders such as charge point operators (CPO), e-Mobility service providers, in addition to OEMs or third party data servers and end-user interfaces [73],

The core EV-related communication protocols can be classified in front-end protocols, between the EV and EVSE, and back-end protocols, between EVSE and a third party such as CPO or aggregator (see Figure 3). EV flexibility requires the development of open (instead of proprietary) and widely-accepted protocols that support the advanced communication requirements of smart charging and V2G while keeping high standards for data security and privacy. However, recent smart charging implementations did not have access to relevant charging session information such as SoC or battery sizes, limiting smart charging algorithms (see [74] 75]), and currently there are multiple competing protocols without full V2G support. Upcoming releases of open communication protocols, such as ISO 15118-20 standard (between EVSE and EV), and OCPP 2.0 or IEC 63110 (between EVSE and aggregator or CPO), will allow advanced communication between different stakeholders, including V2G support, and garner broad industry support [71][76]. While smart charging is already possible for most EVs, widespread commercialization of V2G-ready EVs and EVSEs is expected only for $2025[77]^{2}$ For an in-depth discussion of EV-related protocols, see [73] and [71].

ICT requirements may differ for different smart control services. Frequency regulation services require high-resolution frequency measurements and low latency communication to be

\footnotetext{
${ }^{2}$ Currently, only EVs using CHAdeMO are V2G-compatible, which are less common in Europe [78.
} 


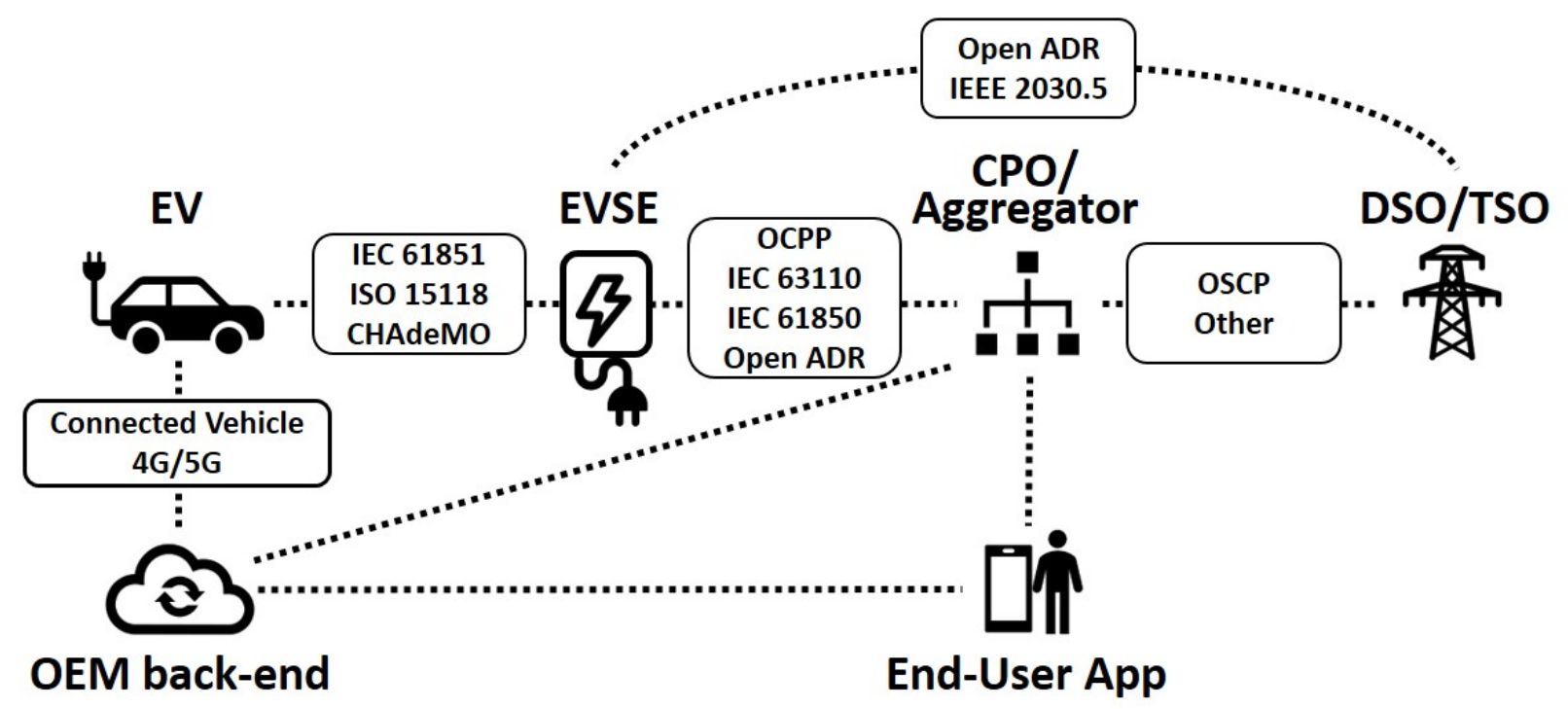

Figure 3: Main communication links for flexibility services, based from [73] and [7]

able to respond according to frequency dynamics. This may not be the case for distributionlevel congestion management, as thermal dynamics work in slower timeframes. Coordination schemes also affect communication requirements: centralized schemes require greater data exchange between the central controller (aggregator or other stakeholder) while decentralized control schemes work with less information exchange and may distribute computational capabilities.

Stakeholders should aim to identify the ICT requirements to monitor and control flexible sources in the grid, and work for common and open source ICT protocols and standards suitable for smart charging and $\mathrm{V} 2 \mathrm{G}$.

Table 1 overviews the main technical requirements for flexibility services.

\section{Economic aspects}

\subsection{Flexibility procurement framework}

EV flexibility today is exploited commercially by aggregators in existing markets, i.e. energy management and system balancing [43] [79]. However, there is still no widely accepted framework for the use of flexibility at distribution level. Different frameworks have been proposed in the literature and in demonstrator projects. According to CEER, models for flexibility procurement by DSOs can be divided in the following categories [80]: 
Table 1: Technical requirements of EV flexibility services

\begin{tabular}{ccccc}
\hline Customer & Service & Charger capability* & Duration & ICT** \\
\hline \multirow{3}{*}{ End-User } & Bill optimization & SC & Hours & Smart meter, HEMS \\
& Self-consumption & SC, V2X & Hours & HEMS \\
& Back-up power & V2X & Hours, Days & None \\
\hline \multirow{2}{*}{ DSO } & Voltage regulation & Q, V2X, SC & Continuous & Low (decentralized) \\
& Congestion management & V2X, SC & 15 min to 2h & Medium (DSO-Agg-EVSE) \\
& Fault-restoration & V2X & 30min to 3h & Medium (DSO-Agg-EVSE) \\
\hline \multirow{2}{*}{ BRP/TSO } & Balancing & V2X & 1-15min & High \\
& Energy Arbitrage & SC & 15min-hours & Depending on coordination \\
\hline
\end{tabular}

* SC: Smart charging (unidirectional). V2X: bidirectional charging. Q: reactive power compensation.

**Agg: Aggregator. HEMS: Home Energy Management System

\subsubsection{Rules-based approach}

This refers to grid codes that define the technical requirements for grid connection. They are used mainly to maintain the security and stability of the electricity grid, such as disconnection under fault conditions or harmonic distortion limits.

Imposing smart charging or V2G through grid codes might not be viable, as it may impose a barrier to the development of market-based flexibility services. However, reactive power compensation for voltage regulation (Volt-VAr regulation) could be made a requirement for V2G-capable EVs, as it is already a requirement for power injecting DER (such as PV panels) in some countries [64] 81] and is supported by the IEEE 1547 Standard [82]. It has also been proposed as a connection requirement for unidirectional EV charging infrastructure [81] [55], though it may unfairly burden grid access for EVs, as other loads do not have to comply with this requirement, and may prove a barrier to the technological development of innovative services (like dynamic reactive power control).

Grid codes are defined at a regional or national level. While many aspects are shared across countries, there are still differences among requirements and varied treatment for emerging technologies, such as storage and EVs [83]. Compliance with diverse national grid codes may present a barrier for the massive deployment of V2G-capable EVs by OEMs with international presence, as well as creating issues with EVs that can move across national or regional borders (see Section 5.1.2). 


\subsubsection{Connection agreements}

In this arrangement DSOs work with customers to form an agreement for the provision of flexibility. Connection agreements have been successfully implemented for congestion management using smart connections for renewable generation in Europe [84 85] 86]. Generators with a smart connection are interruptible, which means they can be (partially) curtailed if there is local congestion, but benefit from lower connection costs and shorter delays. Arrangements for EV charging infrastructure can take two approaches: interruptible contracts or variable capacity contracts (VCC).

Interruptible contracts, similar to those for renewable generation, can (partially) curtail EV charging infrastructure according to system conditions. This type of contract leaves direct control of the EV charging process to DSOs, and presents the risk of precluding EV mobility needs, thus potentially meeting with lower user acceptance. My Electric Avenue project tested an interruptible solution where a system temporarily curtailed EV charging to respect the limits on the local grid infrastructure [12].

VCCs provide the customer with a variable maximum power they can withdraw from the grid according to a schedule (either fixed or dynamic) set by the DSO, while benefiting them with lower network tariffs. For example, users can have a reduced maximum capacity during peak load hours but an increased maximum capacity during off-peak hours [87, as shown in Figure 4. This type of contract has recently been proposed for residential users in Spain, where customers can choose a higher subscribed capacity for off-peak hours (from midnight to $8 \mathrm{AM})[88$.

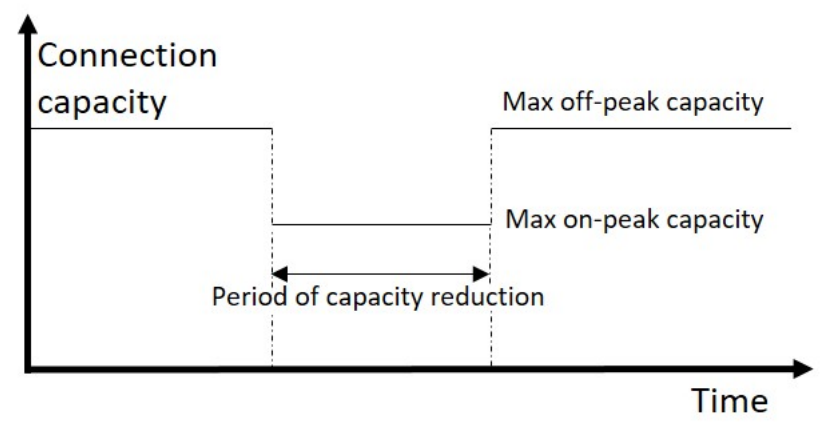

Figure 4: Example of variable capacity contract. Adapted from 87 
A VCC solution for EVs was demonstrated in the FlexPower project in Amsterdam for PCI. In this project, PCIs have a reduced charging capacity during the evening peak load time but benefit from increased capacity during the rest of the day, thus reducing congestion and optimizing EV charging times during the rest of the day. Results have shown minimal impact for users who charge during peak hours while increasing the energy charged by users who charge during off-peak hours [89]. In the Electric Nation project an aggregator-based VCC was implemented. The aggregator managed the charging process of fleets of over 250 EVs to follow a capacity limit curve provided by the DSO, that emulated the spare capacity on a MV feeder [74]. A similar aggregator-based solution was implemented for PCIs in one of the Invade project demonstrators [75].

These contracts have been proposed mainly to deal with grid congestion and investment deferral, but they could also be used for local voltage support, for example, by providing faster connections for EV charging infrastructure if they provide reactive power compensation.

\subsubsection{Network tariffs}

Network tariffs are used to recover the cost of operating and planning distribution and transmission grids, and they are one component of end-user retail prices along with energy costs and taxes and levies. Network costs represent an average of $25 \%$ of the electricity bill in Europe [90]. Tariffs should reflect the costs of the distribution system, giving incentives to develop of different forms of demand side response mechanisms [91], and they are one of the main levers to incite end-users to adopt smart charging or $\mathrm{V} 2 \mathrm{G}$ strategies to reduce their energy bills in a behind-the-meter fashion.

Network tariff structure can be broken down into energy-based charges (related to the energy consumed by the customer, in $€ / \mathrm{kWh}$ ), capacity-based charges (related to the contracted or maximum power of a customer, in $€ / \mathrm{kW}$ ), and fixed charge (related to each point of connection, in $€$ ), with most countries in Europe presenting a mix of these charges. Differences in network tariffs will impact end-customer DER adoption and EV charging strategies [92] [93]. Furthermore, tariffs can be temporally and geographically differentiated, with different rates for time periods, such as peak and off-peak, or grid zones. 
Energy-based tariffs can depend on when the energy is consumed by the customer, such as Time-of-Use (ToU) or critical peak pricing tariffs, which provide lower prices during offpeak hours and higher prices during peak hours. ToU tariffs have been widely studied in the EV smart charging literature. Most studies conclude that simple two- or three-rate tariffs (on-peak, off-peak and super-off-peak) can reduce congestion issues in distribution grids in low EV penetration scenarios, by shifting the charging process to off-peak hours. However, in high-penetration scenarios they can create even higher peaks due to a synchronization of EV charging at off-peak periods [94][55] [95], or with other controllable loads such as electric water heaters or heat pumps [96].

Retail tariffs can reflect both network and energy price signals with high temporal granularity. Dynamic electricity tariffs linked to (sub)-hourly wholesale electricity prices are already practised in several countries in Europe, such as Spain, Norway, 97] and the UK 98. However, this type of dynamic pricing can still present high load peaks from EVs as they concentrate the charging process on low-price hours [99] and may not necessarily correlate to local congestion periods in the distribution grid. This is especially true in high-renewable systems, where wholesale electricity prices are not correlated to total (or local) demand [95], thus potentially further increasing the need for distribution network reinforcements [48]. Thus, challenges arise if only system-wide signals are passed to end-user tariffs.

Currently, significant research is being carried out on distribution locational marginal prices (DLMPs) [100] 101] 102] [103, where the price of electricity reflects the costs of producing and delivering electricity at each node of the distribution grid (nodal marginal pricing). Depending on the formulation, DLMP-based frameworks account for line capacities, voltage limits, active losses and even reactive power. These frameworks can reflect the distribution grid conditions, but they are also difficult to implement in practice, especially in Europe where most countries do not even use nodal prices at the transmission level. Note that DLMPs can create issues for the transparency and stability of end-user tariffs [9][104]. Also, they can go against the equalization principles that exist in network tariffs in some European countries, like France [105] or the Netherlands[106], as DLMPs can make customers connected in weaker, more congested grids, experience higher grid tariffs.

Capacity-based tariffs charge customers according to their contracted capacity or their 
maximum demand during a given period (demand charges). With this type of tariffs, users are given the incentives to adopt strategies to reduce their maximum consumption. If demand charges are computed at the peak-load period (coincidental demand charges), they can help reduce congestion in the distribution grid [93]. However, capacity tariffs can pose other problems, such as creating a barrier to the development of fast-charging stations that may be required to ensure EV adoption [107].

Currently, most network tariffs respond to system-wide criteria, such as system-wide peak load. This is shown in Table 2, detailing network tariffs for residential customers in selected countries in Europe (end-users tariffs need to account for energy costs and taxes and levies as well). Most countries have static network tariffs, with fixed on-peak/off-peak periods throughout the year and no geographical differentiation. It is thus necessary to develop tariffs with higher temporal and geographic granularity that can provide signals for a better utilisation of distribution grids [108] [91] [104]. For example, [109] proposed a network tariff for Spain based on compounded peak/off-peak tariffs at the different voltage levels, thus reflecting both transmission and distribution constrained periods. Likewise, New York utility ConEdison tested a residential retail tariff that considered dynamic day-ahead electricity prices for the energy component plus both transmission and distribution coincidental peak charges independently for network charges [110]. Network tariffs can provide incentives to end-users to reduce congestion in the distribution grid, but they might be less suitable for other flexibility services like voltage regulation or phase balancing in LV grids.

Table 2: Network tariffs for residential customers in selected countries ( $\leq 10 \mathrm{~kW}$ or equivalent).

\begin{tabular}{llllll}
\hline Country & Tariff type & E. type* & Cap. type* $^{*}$ & Locational signal & Temporal signal \\
\hline France [105] & E + C + F & Seasonal ToU (2) & Subscribed cap. & Local (substation level) & Static \\
UK [11] & E + C + F & ToU (3) & Subscribed cap. & Uniform within DSO & Static \\
Germany [12] & $\mathrm{E}$ & Flat & - & - & - \\
Netherlands [106] & $\mathrm{C}$ & - & Subscribed cap. & - & - \\
Belgium [113] & $\mathrm{E}$ & ToU (2) & & Uniform within DSO & Static \\
Spain [88] & $\mathrm{E}+\mathrm{C}+\mathrm{F}$ & Seasonal ToU (2) & Subscribed cap. & Uniform within DSO & Static \\
\hline
\end{tabular}

E: Energy, C: Capacity, F: Fixed, ToU: Time-of-Use

* Parenthesis indicates the number of pricing periods. 


\subsubsection{Market-based}

In this approach, DSOs explicitly procure flexibility services from a market, either via long-term bilateral contracts or via a short-term market platform. This approach is preferred by regulators [80].

Bilateral contracts can enable flexibility procurement for medium- to long-term horizons. In this case, DSOs identify in advance the flexibility requirements enabling to defer or avoid costly reinforcements or improve grid operation (for planned maintenance or faultrestoration) and procure flexibility through long-term contracts. This type of contract can be signed between DSOs and flexibility providers after a tender process, or through overthe-counter contracts if there are no sufficient conditions for market formation.

This approach has been adopted by the UK's DSOs. UKPN, the London-area DSO, adopted a "flexibility first" policy towards all new investments in MV and HV (over $10 \mathrm{kV}$ ). They have identified grid sections where the use of flexibility during certain critical periods (usually in winter, during peak load) could help defer reinforcements, and have subsequently organized tender processes to procure flexibility from distributed sources since 2018. This market is supported by the flexibility platform PicloFlex, for which Figure 5 gives a screenshot depicting the flexibility requirements for a tender zone. Similar processes have been led by the other UK's DNOs [114] and by French DSO Enedis [115], in an effort to contract flexibility for services including congestion management for investment deferral, reactive power compensation and fault-restoration services. Similarly, New York state utilities are using request for proposals for investment deferral though their non-wire alternatives (NWA) programs [116].

Flexibility trading within shorter timeframes, such as day-ahead or intraday, have been proposed in the literature and in various demonstrator and pilot projects in the form of flexibility market platforms and local energy markets.

The first commercial implementations of short-term local flexibility trading are Enera

(Germany) [118 and GOPACS (Netherlands) [119], where DSOs and TSOs procure flexibility to manage RES-driven congestion, supported by existing energy trading market platforms (EPEX Spot for Enera and ETPA for GOPACS). EV aggregators participating in these market platforms can provide flexibility to DSOs by offering location-specific bids. 


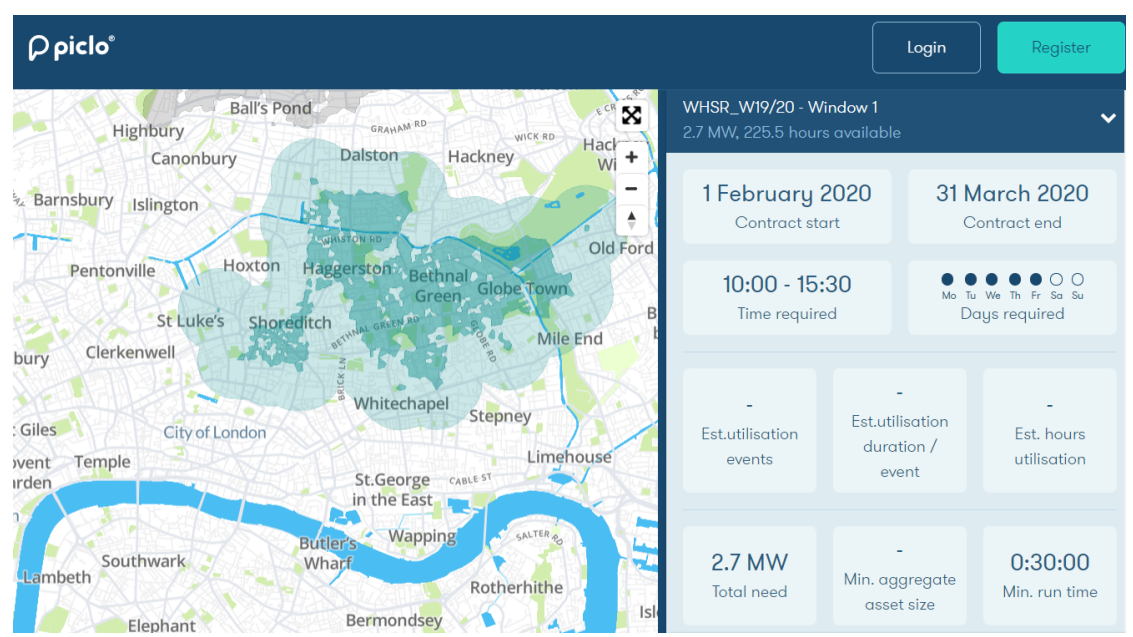

Figure 5: Screenshot of the PicloFlex flexibility platform, showing the flexibility requirements during winter 2020 in the London Area. [117]

The INVADE demonstrator project developed a flexibility management platform to control batteries and EVs to support higher penetration of renewable energy. Via this platform flexibility services are proposed for end-users (behind-the-meter services), DSOs (congestion management and voltage regulation) and BRPs (energy arbitrage) [120]. Likewise, the Interflex project studied flexibility provision by EV aggregators at the LV level implementing a day-ahead and intraday local flexibility market in its Netherlands demonstrator [121].

On a more local level, local energy markets (LEM) are marketplaces that allow peerto-peer energy and flexibility trading by end-users (prosumers) at community level. By considering distribution grid constraints, LEMs are expected to help balancing the local grid. The EMPOWER project demonstrated a LEM, developing a pool-oriented platform to buy and sell energy and flexibility at the local level. In this project, a smart energy service provider fulfills the central role of the LEM, by managing the trading platform and acting as an aggregator of end-users assets that can respond to flexibility requests from the DSO and participate in wholesale energy markets [122].

A market-based approach raises the issue of product definition and procurement. For this, flexibility products should be defined, in particular the power (active or reactive), duration and location requirements. A settlement period according to the services and flexibility sources characteristics (ideally close to real-time to consider uncertainty) also have to be defined, along with consumption baselines for flexibility settlement that have to be accepted 
by all stakeholders [22]. Furthermore, the product definition should not pose barriers to entry to distributed flexibility resources, for example by setting over-high bid size thresholds or duration requirements [80] [123].

Local market platforms also require advanced technical capabilities from DSOs, such as demand forecasting with high spatial granularity [124] and LV state estimators to monitor the grid in (near) real-time to activate flexibility [125].

Flexibility platforms have great potential to enable new business models to emerge. However, they are only possible where there are enough participants to create a competitive market, thus making them unsuitable to solve issues in small areas without many flexibility assets, or where a small market size can produce (prohibitively) high transaction costs [80].

Note that multiple alternatives can coexist, like flexibility market platforms, where aggregators would trade end-user flexibility to other electricity system actors (DSOs, BRPs, TSOs), alongside advanced electricity tariffs and flexible contracts that would incite customers to actively manage their consumption. [126] analyzed nine mechanisms for congestion management at the DSO level using EV flexibility, based on combinations of four basic frameworks for flexibility (interruptible contracts, advanced tariffs, VCCs and flexibility markets). Dynamic electricity tariffs coupled with flexible contracts or flexibility markets, are able to solve DSO grid congestion with little to no impact on end-users.

Based on our analysis, Table 3 provides a summary of the value frameworks that can enable the provision of different flexibility services by EVs. Congestion management can be tackled through various solutions, including local flexibility markets and network tariffs. On the contrary, voltage issues require more research to develop flexibility procurement frameworks.

\section{Regulatory aspects}

\subsection{Regulation and Policy}

\subsubsection{DSO roles and responsibilities}

Historically, the DSOs operated radial grids with unidirectional power flows from the transmission grid to end-users, where main concerns (congestion and voltage issues) were addressed by investing in grid reinforcements through a "fit-and-forget" approach. This 
Table 3: Flexibility services to be provided by EVs and the associated value frameworks.

\begin{tabular}{|c|c|c|c|}
\hline Customer & Level & Service & $\begin{array}{c}\text { Value } \\
\text { Framework }\end{array}$ \\
\hline \multirow{2}{*}{ End-User } & \multirow{2}{*}{$\begin{array}{l}\text { Behind- } \\
\text { the-meter }\end{array}$} & Bill optimization & Grid tariffs \\
\hline & & Back-up power & End-user reliability \\
\hline \multirow{4}{*}{$\mathrm{DSO}$} & \multirow[b]{2}{*}{$\mathrm{LV}$} & $\begin{array}{l}\text { Phase unbalance } \\
\text { Voltage regulation }\end{array}$ & $\begin{array}{c}\text { Grid codes } \\
?\end{array}$ \\
\hline & & Congestion management & $\begin{array}{c}\text { Grid tariffs } \\
\text { Flexible contracts } \\
\text { LEM }\end{array}$ \\
\hline & MV & $\begin{array}{c}\text { Voltage regulation } \\
\text { Congestion management }\end{array}$ & $\begin{array}{c}\text { Grid tariffs } \\
\text { Flexible contracts } \\
\text { Flexibility tenders } \\
\text { Local flexibility markets }\end{array}$ \\
\hline & $\mathrm{MV} / \mathrm{LV}$ & Fault restoration & $\begin{array}{l}\text { Bilateral contracts } \\
\text { Flexibility tenders }\end{array}$ \\
\hline TSO/BRP & System-wide & $\begin{array}{c}\text { Balancing services } \\
\text { Energy arbitrage } \\
\text { Imbalance compensation }\end{array}$ & $\begin{array}{l}\text { Balancing markets } \\
\text { Energy markets }\end{array}$ \\
\hline
\end{tabular}


approach was compounded by a regulatory framework that remunerated DSOs based on their capital expenditures (CAPEX), inciting them to invest in costly infrastructure to solve grid issues instead of using operational measures (OPEX).

However, the surge of DERs and digitalization is shifting DSOs roles and responsibilities towards more proactive grid operation [5] [127]. With this approach, flexibility management at local level can provide more efficient use of existing assets, deferring or avoiding the need for costly infrastructure and reducing the risk of stranded assets in the event of uncertain load growth evolution [128]. This requires adapting current grid planning and operation practices to consider flexible assets.

Regulatory frameworks need to evolve to incite DSOs to implement smart and flexible solutions at local level as cost efficiency measures, thus moving beyond a CAPEX-based to a TOTEX-based (total expenditure) framework, with incentives to improve quality of service and innovation. This vision has been set out by a number of stakeholders, from academics [22] [127] [129] and regulators [130] [131] [132], to DSOs themselves [5] [39]. An example of this shift is the UK's performance-based network regulatory framework RIIO (Revenue $=$ Incentives + Innovation + Outputs) that incites DSOs to create value for end-customers instead of just investing in new assets [133].

In particular, the European Clean Energy Package [134] represents an important step in policy evolution as it demands DSOs the active management of flexibility resources, which would significantly reduce network costs and improve reliability. European regulators see future DSOs as neutral market facilitators, similar to the roles taken by TSOs in Europe, enabling different DERs to participate in energy and flexibility markets, at both the local and system-wide level. It also restricts the actions of DSOs, as it bars them from directly owning or operating flexibility resources (including storage and EV charging infrastructure) [80], unless there is a market failure [127].

The need for flexibility management is also acknowledged outside Europe. In the US, California's Smart Grid [135] and New York's Reforming Energy Vision [136] initiatives have pushed utilities to adopt DERs in their operation and planning phases and implement NWAs as an alternative to grid investments [137].

The regulatory framework in the US, with vertically integrated utilities, allows utilities 
to have ownership and direct control of DERs, as well as direct contact with end-customers. However, their roles and responsibilities are evolving, with different views on the future utility, from expanding their roles to provide more grid and customer services, to setting them as market facilitators (like unbundled DSOs in Europe), or even completely separating the role of grid ownership from operation and planning (Independent DSO model) [135] [138] [139]

\subsubsection{EV status in the grid}

V2G-able EVs face great difficulties regarding their connection requirements and legal status as flexibility providers. Connection requirements can be burdensome, as V2G-capable EVs need to comply with requirements both as producers and consumers, as well as administrative procedures to declare and allow distributed sources to participate as flexibility providers. Legal status of V2G installations should also be clarified and aligned with that of storage, with tariffs and charges that prevent double taxation.

Regulators, system operators, and EV and EVSE manufacturers need to work to standardize interconnection requirements to ensure system and end-user safety, while easing administrative procedures. For example, the French regulator issued a series of recommendations regarding the interconnection requirements, mainly for the definition of the decoupling protection, as well as simplification of administrative procedures [140]. In 2019, Delaware state passed legislation that defined the perimeter of V2G, defined clear interconnection procedures (adopting SAE J3072 safety for on-board bidirectional chargers [141]) and allowed net-metering to provide a level-playing field with utility-scale storage [142]. These measures have been suggested to other states as well [143].

\subsection{Interactions with grid operators}

An important aspect is how the different stakeholders interact along the flexibility value chain. There are interactions between flexibility providers and flexibility customers, in this case EV users and DSOs respectively, and interactions between DSOs and TSOs as potential flexibility customers, where their level of coordination and cooperation will affect how local flexibility is used. 


\subsubsection{EV users-DSO interaction}

DSOs can procure flexibility from end-users directly or indirectly. As mentioned in Section 5.1. DSOs can procure flexibility using different solutions. By using direct obligations (grid codes) for flexibility provision or contract arrangements (such as interruptible contracts), DSOs will directly interact with EV users acquiring permission to directly control the EV charging process.

On the other hand, market-based procurement via flexibility platforms usually needs an aggregator that would gather multiple flexibility resources. This is currently the case for ancillary services and BRP energy arbitrage as done by existing EV aggregators. It could be expected that a growing number of EVs will become associated to an aggregator's program, therefore likely to meet communication and control requirements for the smart charging process. This will allow the provision of market-based flexibility services to DSOs by EV fleets.

\subsubsection{DSO-TSO interaction}

Currently there is only limited cooperation between DSOs and TSOs. As more DERs are connected into distribution networks and start providing ancillary services, like EVs providing frequency response, DSO-TSO cooperation will become increasingly important to guarantee the safe and reliable operation of the power system. This has been highlighted by the scientific community [84] [144], industry and regulators [80], and was considered as a key aspect in the European Clean Energy Package.

SmartNet is a key demonstrator project that focused on DSO-TSO coordination, considering data exchange, monitoring and the provision of ancillary services from distributed sources [144]. Five possible coordination schemes for flexibility procurement by DSOs and TSOs were analyzed and different schemes emerged depending on the level of DSO-TSO cooperation, their roles and responsibilities definition and the level of integration of markets (centralized or decentralized). Higher coordination can present benefits on operational security and reliability and in asset efficiency, both in centralized and decentralized schemes, but it also carries higher computational and ICT burden, and poses regulatory issues.

In [84], authors analyzed possible cooperation between DSO and TSO according to the 
system state for congestion management, considering both operational issues and market issues. They found that cooperation can arise from forward stages (long- and medium- term), by harmonizing practices and data for capacity calculation, at day-ahead (short-term), by joint or coordinated flexibility procurement, and in real-time stages, by ensuring grid security in firmness and capacity allocation. Conflicts may arise if flexible resources (such as EVs) are required by the DSOs and TSOs concurrently. [47] analyzed this issue in a context of distribution congestion management and primary frequency response services. Definition of priorities on flexibility procurement, activation and compensation will be needed in these cases.

\section{End-user aspects}

\subsection{User behavior}

To evaluate the impacts and flexibility potential of EVs it is necessary to have reliable data on user behavior and advanced models to forecast EV usages and electricity demand [52] 145] 146]. In particular, the key aspects to factor in are energy requirements and connection time, which depend on how EVs are used, and when, where and how much they are charged. Since EVs are a relatively new technology, this data is scarce or might not be representative of future trends. This make demonstrator projects hugely valuable since they provide insight into real data on EV usage and charging patterns. For example, My Electric Avenue [147] [148], SwitchEV [149] and Electric Nation [74] projects provide insights on residential EV user behavior in the UK, showing the behavioral diversity of EV drivers. Driving and charging patterns are affected by various factors, including driving purposes (commuting or other purposes), driving behavior (aggressive drivers have lower energy efficiency), driving conditions (topography, road congestion, outside temperature) [149]), plug-in preferences, battery sizes (EVs with bigger batteries tend to be connected fewer times per week) [52], and availability of charging infrastructure.

Flexibility provision will also depend on the use-case and charging infrastructure considered. At-home (overnight) charging has been widely studied [44][149] [55] [94] [99], showing to have high flexibility potential. Studies analyzing charging patterns in PCI [150][151], 
and in workplace locations [152], have also found that EVs are idle (not charging) a significant amount of time, thus inducing inefficiencies in charging infrastructure utilization, but also opening an opportunity for smart charging. Therefore, understanding EV user driving and charging patterns is necessary to properly assess the impacts of EV integration and to identify opportunities for flexibility provision.

Understanding behavioral aspects is more important at distribution level, as clustering of EV users into a certain area or neighborhood can create issues at local level, even while overall EV diffusion remains low [153][154]. Usage patterns at local level (urban vs. rural usage), plug-in behavior [155] [52], and weekly and seasonal variations in EV usage [145] will also impact the distribution grid and the flexibility potential of EV fleets.

EV user segmentation is also important to identify fleets with high flexibility potential. Identifying different types of usages/users can provide complementary sources of flexibility and should enable aggregators to better design flexibility offers around end-users needs. For example, the driving and charging patterns of commercial fleets are different than private ones [156]. Commercial or company fleets, with predictable and consistent usage patterns, can be better assets for flexibility provision [157]. Demonstrator projects like Parker [42] and GridMotion [43] have used company fleets to demonstrate V2G-based grid services.

\subsection{User acceptance}

Mobility is and will continue to be the primary purpose of EVs, so flexibility services will need to meet end-user mobility requirements and expectations. From a technical perspective, flexibility services should guarantee a sufficient range to fulfill travel requirements, and should not induce significant aging in the EV equipment, especially the battery, while also enabling users to retain control of the charging process (opt-out). My Electric Avenue project implemented a local congestion management trial in a residential and workplace environment. They found that end-users opted out of controlled charging when the control process started to affect their charging requirements. Likewise, the Electric Nation project showed that opt-out option was key for customer satisfaction, though rarely used (less than $5 \%$ of charging events) [74].

Providing economic incentives can help the acceptance of controlled charging, however 
they are not sufficient nor mandatory. [158] conducted a survey on acceptance of night-time utility-controlled charging in Canada, and found potential support from between a half and two thirds of the population and economic incentives increased readiness to accept smart charging. Main concerns were loss of control and privacy. However, [159] found that economic incentives are expected, though not significant for acceptance, and that the main factor is an understanding for the need for grid flexibility and RES integration. In a more general case, the EMPOWER project found that key factors for end-user acceptance of flexibility services and local energy markets are: first, consuming local and renewable energy, and second, the financial incentives that can be gained (electricity costs reduction) [160].

Finally, awareness and knowledge of grid services, specially V2G, is still very low, even among experts [57]. It is therefore critical to raise awareness and educate EV users about the utility of flexibility services to support the local grid and increase renewable energy penetration, while developing services around end-users needs and expectations. This shows that while economic incentives can help to increase user acceptance, other options such as raising awareness, sharing charging data and even gamification [161] can boost end-user engagement.

\section{Discussion}

EV grid integration is one of several challenges that face electricity systems. However, it presents a great opportunity to move towards distributed, decarbonized, flexible power systems. Exploiting EV flexibility can provide benefits for different actors in electricity systems, from supporting the grid by providing ancillary services and increasing renewable energy penetration, to reducing end-user electricity bills and providing energy autonomy as back-up power. In particular, for distribution networks, EV flexibility can help to defer or avoid costly reinforcements in highly uncertain scenarios, thus reducing the risk of stranded assets. Furthermore, EV flexibility can help to make more efficient use of existing infrastructure, by providing peak shaving services and voltage support, and by providing fault restoration or islanding services to reduce non-served energy.

The technical impacts of EV diffusion and the benefits of charging flexibility for distribution grids have been widely studied in the research community. These studies have 
mostly focused on developing new control algorithms and architectures for EV fleets, and have tested them in different use cases, most of them centered on LV residential grids. There has been less research on MV grids and rural or industrial/commercial environments.

The flexibility capability of EVs has been proven from a practical perspective. Demonstrator projects have successfully managed to provide system-wide services such as primary frequency response in the US (University of Delaware, PJM Interconnection) and in Europe (Parker, Denmark and GridMotion, France), and there are already EV aggregators that have commercial offers on the market, such as Nuvve and Jedlix. These actions show that communication and control of large EV fleets is possible.

There are, nevertheless, some technical barriers to the development of flexibility services at the distribution level. The main technical barriers are:

Observability in distribution grids: Need for increased observability by DSOs, ideally close-to-real-time, to enable forecasting and flexibility activation.

Battery aging: Battery aging is a major factor in the economic viability and user acceptance of grid services. This effect should be thoroughly studied in the context of distribution grid services, which can be more energy-intensive than frequency response services.

Charging technology: Bidirectional chargers are still not a mature technology. They remain expensive and round-trip efficiency can be an issue. However, costs are expected to fall and they could include additional capabilities, such as reactive power compensation.

However, the main barriers to develop EV flexibility are not technical but economic and institutional. These are:

Active management of distribution grids: DSOs should move from a "fit-and-forget" approach towards proactive management of their grids. This includes DSOs developing new roles and responsibilities for grid operation and planning (including forecasting and grid observability), as well as putting in place mechanisms that procure flexibility in a cost-efficient way. Regulators need to work on providing DSOs with the incentives for innovation and cost-efficiency. 
EV status in the grid: V2G-able EVs face significant regulatory and technical burden to provide flexibility. Simplification and standardization of connection procedures and adapted metering options are recommended.

DSO-TSO cooperation: Need for increased cooperation and coordination to enable flexibility coming from all levels of the grid and to maintain secure and reliable operation of the power system.

Value frameworks for flexibility: The mechanisms for exploiting flexibility at the distribution level remain limited, but they are emerging, both in demonstrator projects and real-life implementations like flexibility tenders and NWAs.

Value of services: Since the use of flexibility at the distribution level is just emerging, the value that can be extracted from providing flexibility is still unclear. The additional value that V2G can provide with respect to smart charging still needs more research.

However, in recent years there have been several advances in this regard. Regulators have shown increasing interest in exploiting local flexibility from different sources and encouraging smart management of DERs. The European Commission Clean Energy Act made a first step, acknowledging the benefits of flexibility to reduce network costs and the need for regulators to introduce incentives to prompt DSOs to better manage and plan their grids and foster innovation. Note that European regulators (CEER) have also addressed the evolution of DSO roles, the associated incentives, and the use of local flexibility.

Considering EV flexibility, regulators like OFGEM (UK) and CRE (France) have seriously taken on the task of analyzing the challenges of EV integration (see [162] [140]), and several V2G demonstrator projects have been funded, especially in the UK [163]. The Netherlands has run several DSO-led projects where they test new solutions for hosting EVs, with active involvement of DSOs through Elaad, an association for EV development.

In this regard, demonstrator projects play a crucial role by addressing all four key aspects: technical, economic, regulatory and user-related. Table 4 summarizes the analyzed demonstrator projects in smart grids and VGI.

Most VGI projects have focused on proving the technical feasibility of EV flexibility and 
Table 4: Demonstrator projects analyzed

\begin{tabular}{|c|c|c|c|}
\hline Project & Period & Region & Research Topic \\
\hline evolvDSO & 2013-2016 & Europe & $\begin{array}{l}\text { DSO future roles, } \\
\text { tools and methods }\end{array}$ \\
\hline SmartNet & $2016-2018$ & Europe & DSO-TSO coordination \\
\hline IDE4L & $2013-2016$ & Europe & $\begin{array}{l}\text { Automation and ICT } \\
\text { for distribution grids }\end{array}$ \\
\hline EMPOWER & $2015-2017$ & Norway & Local energy markets \\
\hline INVADE & 2017-2019 & Europe & $\begin{array}{c}\text { Distributed storage management } \\
(\mathrm{EV} \& \mathrm{BESS})\end{array}$ \\
\hline InterFlex & 2017-2019 & Europe & $\begin{array}{l}\text { Flexibility for distribution grids } \\
\text { Local flex markets }\end{array}$ \\
\hline BienVEnu & 2016-2018 & France & $\begin{array}{l}\text { Smart charging } \\
\text { in residential buildings }\end{array}$ \\
\hline Parker & 2016-2018 & Denmark & $\begin{array}{l}\text { Grid services using } \\
\text { commercial EVs }\end{array}$ \\
\hline ACES & 2017-2019 & Denmark & Vehicle-Grid Integration \\
\hline My Electric Avenue & $2013-2015$ & UK & $\begin{array}{l}\text { EV integration } \\
\text { in LV grids }\end{array}$ \\
\hline Electric Nation & 2017-2019 & UK & $\begin{array}{l}\text { Large scale } \\
\text { Smart Charging trial }\end{array}$ \\
\hline Electrific & 2017-2019 & Europe & $\begin{array}{l}\text { Solutions for e-Mobility } \\
\text { (including VGI) }\end{array}$ \\
\hline GridMotion & $2018-2020$ & France & EV Grid Services \\
\hline Invent & $2017-2020$ & California & EV Grid Services \\
\hline FlexPower & 2017 & Amsterdam & VCC for PCI \\
\hline
\end{tabular}


creating new technical solutions for EV grid integration without considering value mechanisms (Electrific with a solution for LV voltage and congestion management [164], My Electric Avenue with a solution to handle overloading in LV residential grids), or their economic viability on existing value mechanisms (balancing markets in Parker, balancing markets and energy arbitrage in GridMotion). An exception is the FlexPower project, which has proven the benefits of the VCCs as an innovative mechanism for PCI. Similar solutions could be tested for other services, such as voltage regulation, where charging infrastructure could benefit from lower connection costs or a higher capacity contract if it actively regulates voltage, either using active or reactive power compensation.

Other projects have focused on short-term market solutions, such as flexibility platforms with varying levels of coordination with TSOs (SmartNet, Interflex, INVADE), or local energy markets (EMPOWER). However, this kind of solution is not yet ready to be implemented, as flexibility resources need to become more abundant to allow competition at the local level. For this reason, the first implementations have been in the form long-term contracts (tenders in the UK and France, NWAs in the US).

Table 5 summarizes the services proposed in VGI demonstrator projects and the value framework in which they can be translated.

Future smart grids will feature all four frameworks mentioned. Grid codes are needed to ensure grid reliability, for example by defining requirements for protection under fault conditions, or to reduce power quality issues [11]. The deployment of smart meters will allow the implementation of dynamic tariffs with higher temporal and geographical granularity that will incite users to adopt smart charging strategies, reducing the burden on distribution grids. The implementation of dynamic tariffs should help dealing with most day-to-day grid issues. However, contingencies can still occur in the distribution grid. Flexibility procurement through mid-to-long-term auctions or flexible contracts can improve distribution grid reliability and planning, by ensuring a minimum amount of flexibility available for contingencies or extreme events. In the operational timeframe, flexibility can be procured at lower costs through local flexibility markets in coordination with balancing flexibility for the TSOs.

Research gaps have been identified, which should be further addressed in the literature:

- A thorough characterization of flexibility requirements for distribution grids, i.e., the 
Table 5: Analyzed EV related demonstrator projects.

\begin{tabular}{ccc}
\hline Project & Service & $\begin{array}{c}\text { Value } \\
\text { framework }\end{array}$ \\
\hline BienVEnu & $\begin{array}{c}\text { Residential building } \\
\text { bill optimization }\end{array}$ & Grid tariffs \\
My Electric Avenue & LV CM & Interruptible contract* \\
Electric Nation & MV CM & VCC via aggregator* \\
FlexPower & Peak shaving & VCC for PCI \\
RES integration & Local flexibility \\
InterFlex & LV CM & market \\
INVADE & LV CM & Local flexibility \\
Electrific & Energy arbitrage & market \\
Parker & LV CM and VR & Frequency regulation \\
GridMotion & Energy arbitrage & Balancing markets \\
& Frequency regulation & balancing markets \\
\hline
\end{tabular}

CM: Congestion management. VR: Voltage regulation.

*Value framework not explicitly addressed in the project 
frequency, duration and amount of flexibility needed to solve grid issues. The models to provide flexibility will differ according to whether the service is required only a few days a year to cope with peak demand, or if it is needed all-year-round. It can also affect the fleet size required to provide these flexibility services and the impact on battery degradation.

- Quantification of the value of different EV services. As most studies analyze each service separately (congestion management, voltage regulation, losses reduction), there is no common valuation of these services. This can help prioritize the services that EVs can provide.

- Quantification of the value of $V 2 G$ with respect to smart charging. While this has been addressed in the frequency response framework (for example in [13]), it has been less thoroughly reviewed at distribution level.

- The coordination of EV flexibility provision within different frameworks still needs further research. For example, the coordination of flexibility provision through market frameworks in the presence of dynamic tariffs for end-users, or the coordination between EVs providing ancillary services to the TSO and to the DSO.

- Studies should consider realistic driving and plug-in behavior. Most studies assume that EVs are plugged-in every day, which overestimates the potential flexibility that EV fleets can effectively provide, and do not consider local mobility patterns (urban vs. rural conditions). Advanced data-driven models can help overcome these issues.

\section{Conclusion}

This work analyzed the technical, economic, regulatory, and user-related aspects that arise for the proactive integration of EVs into distribution grids. This integration takes place against a broader backdrop of cross-sector electrification and decentralized generation that poses serious challenges to distribution grids operation and planning. EVs, through smart charging and V2G, can provide flexibility to electricity systems, thus reducing the impact of their integration and even creating value for different stakeholders along the value 
chain, such as end-customers, aggregators and system operators, and help the integration of renewable energy.

EVs can provide DSOs with various services, including investment deferral on the planning timeframe, and congestion management, voltage regulation and back-up power in operational time frames. The ability to provide these services has been proven technically, but the technologies (bidirectional chargers, reactive power control) and communication protocols needed to exploit the full potential of EV flexibility are not yet widespread.

The main barriers for EVs to provide flexibility services to distribution grids are economic and institutional. DSOs have only recently started to change operational and planning practices to move from a "fit-and-forget" approach to an active management of their grids, so there are no widespread value frameworks under which EVs can provide flexibility to DSOs. This work analyzed four possible value frameworks (grid codes, connection agreements, tariffs and market platforms) to use flexibility at the distribution level, and their applications with $\mathrm{EV}$ fleets in demonstrator projects. In future smart-grids, we expect that these frameworks will coexist, such as dynamic tariffs for EVs that can incite EV-users to charge at low-impact hours, market platforms that can explicitly procure flexibility for DSO needs, and long-term contracts to provide flexibility in the case of unexpected events.

The adoption of smart charging and V2G will ultimately depend on end-user acceptance. The flexibility services covered in this paper will only be possible if they are built around end-users primary need of mobility. Understanding users' mobility and charging habits will be key for flexibility development.

\section{CRediT authorship contribution statement}

Felipe Gonzalez Venegas: Conceptualization, Methodology, Investigation, Writing Original Draft, Writing - Review \& Editing. Marc Petit: Conceptualization, Writing Review \& Editing, Supervision, Funding Acquisition. Yannick Perez: Conceptualization, Writing - Review \& Editing, Supervision, Funding acquisition. 


\section{Acknowledgement}

This research benefits from the support of the Chair "Hybrid Technologies and Economy of Electromobility", so-called Armand Peugeot Chair, led by CentraleSupélec and ESSEC, and sponsored by Stellantis, and the financial support of the ANRT for the CIFRE contract $n^{\circ} 2018 / 0031$.

\section{References}

[1] L. Noel, G. Zarazua de Rubens, J. Kester, B. K. Sovacool, Beyond emissions and economics: Rethinking the co-benefits of electric vehicles (EVs) and vehicle-to-grid (V2G), Transp. Policy 71 (2018) 130-137. doi:10.1016/j.tranpol.2018.08.004.

[2] International Energy Agency, Global EV Outlook 2020, Tech. rep., International Energy Agency (2020).

[3] U. Tietge, P. Mock, J. Dornoff, CO2 emissions from new passenger cars in the European Union: Car manufacturers' performance in 2018, Tech. rep., International Council for Clean Transportation (2018).

URL http: //eupocketbook .orgwww . theicct.org

[4] EV-Volumes - The Electric Vehicle World Sales Database [Accessed: 2021-03-16]. URL https : //www .ev-volumes.com/

[5] E. Rivero, M. Sebastian-Viana, J. Stromsather, A. Ulian, The evolvDSO Project: Key Services for the evolution of DSO's roles, in: 23rd Int. Conf. Electr. Distrib., Lyon, 2015, pp. 15-18.

[6] CENELEC, EN 50160:2010 Voltage Characteristics of electricity supplied by public electricity networks (2010).

[7] M. Neaimeh, R. Wardle, A. M. Jenkins, J. Yi, G. Hill, P. F. Lyons, Y. Hübner, P. T. Blythe, P. C. Taylor, A probabilistic approach to combining smart meter and electric vehicle charging data to investigate distribution network impacts, Appl. Energy 157 (2015) 688-698. doi:10.1016/j.apenergy.2015.01.144. 
[8] C. Protopapadaki, D. Saelens, Heat pump and PV impact on residential low-voltage distribution grids as a function of building and district properties, Appl. Energy 192 (2017) 268-281. doi:10.1016/j. apenergy.2016.11.103.

[9] C. Eid, P. Codani, Y. Perez, J. Reneses, R. Hakvoort, Managing electric flexibility from Distributed Energy Resources: A review of incentives for market design, Renew. Sustain. Energy Rev. 64 (2016) 237-247. doi:10.1016/j.rser.2016.06.008.

[10] G. Pasaoglu, D. Fiorello, A. Martino, L. Zani, A. Zubaryeva, C. Thiel, Travel patterns and the potential use of electric cars - Results from a direct survey in six European countries, Technol. Forecast. Soc. Change 87 (2014) 51-59. doi:10.1016/j.techfore. 2013.10 .018$.

[11] EDSO, Smart charging: integrating a large widespread of electric cars in electricity distribution grids, Tech. rep., EDSO (2018).

URL https://www.edsoforsmartgrids.eu/wp-content/uploads/ EDSO-paper-on-electro-mobility-2.pdf

[12] EA Technology, My Electric Avenue Project Close-Down Report, Tech. rep., My Electric Avenue Project (2016).

[13] O. Borne, Y. Perez, M. Petit, Market integration or bids granularity to enhance flexibility provision by batteries of electric vehicles, Energy Policy 119 (2018) 140-148. doi:10.1016/j.enpol.2018.04.019.

[14] Q. Hoarau, Y. Perez, Interactions between electric mobility and photovoltaic generation: A review, Renew. Sustain. Energy Rev. 94 (2018) 510-522. doi:10.1016/j. rser.2018.06.039.

[15] Everoze, EVConsult, V2G Global Roadtrip: Around the World in 50 Projects, Tech. Rep. October (2018).

[16] G. Fitzgerald, J. Mandel, J. Morris, H. Touati, The Economics of Battery Energy Storage: How multi-use, customer-sited batteries deliver the most services and value 
to customers and the grid, Tech. rep., Rocky Mountains Institute (2015).

URL http://www.rmi.org/electricity_battery_value

[17] J. García-Villalobos, I. Zamora, J. San Martín, F. Asensio, V. Aperribay, Plug-in electric vehicles in electric distribution networks: A review of smart charging approaches, Renew. Sustain. Energy Rev. 38 (2014) 717-731. doi:10.1016/J.RSER.2014.07.040.

[18] J. Hu, H. Morais, T. Sousa, M. Lind, Electric vehicle fleet management in smart grids: A review of services, optimization and control aspects 56 (2016) 1207-1226. doi:10.1016/j.rser.2015.12.014.

[19] K. M. Tan, V. K. Ramachandaramurthy, J. Y. Yong, Integration of electric vehicles in smart grid: A review on vehicle to grid technologies and optimization techniques 53 (2016) 720-732. doi:10.1016/j.rser.2015.09.012.

[20] C. Peng, J. Zou, L. Lian, Dispatching strategies of electric vehicles participating in frequency regulation on power grid: A review, Renew. Sustain. Energy Rev. 68 (2017) 147-152. doi:10.1016/j.rser.2016.09.133.

[21] R. Fachrizal, M. Shepero, D. van der Meer, J. Munkhammar, J. Widén, Smart charging of electric vehicles considering photovoltaic power production and electricity consumption: A review, eTransportation 4 (2020) 100056. doi:10.1016/j.etran.2020. 100056.

[22] K. Knezović, M. Marinelli, A. Zecchino, P. B. Andersen, C. Traeholt, Supporting involvement of electric vehicles in distribution grids: Lowering the barriers for a proactive integration, Energy 134 (2017) 458-468. doi:10.1016/j.energy.2017.06.075.

[23] A. T. Procopiou, J. Quiros-Tortos, L. F. Ochoa, HPC-Based Probabilistic Analysis of LV Networks with EVs: Impacts and Control, IEEE Trans. Smart Grid 8 (3) (2017) 1479-1487. doi:10.1109/TSG.2016.2604245.

[24] T. Li, S. H. Low, A. Wierman, Real-time Flexibility Feedback for Closed-loop Aggregator and System Operator Coordination, in: e-Energy 2020 - Proceedings of the 
11th ACM International Conference on Future Energy Systems, ACM, New York, NY, USA, 2020, pp. 279-292. doi:10.1145/3396851.3397725.

[25] P. Codani, Integration des véhicules électriques dans les réseaux électriques : Modèles d'affaire et contraintes techniques pour constructeurs automobiles, Ph.D. thesis, Université Paris Saclay (oct 2016).

URL http://www . theses.fr/2016SACLC073

[26] K. Knezovic, S. Martinenas, P. B. Andersen, A. Zecchino, M. Marinelli, Enhancing the Role of Electric Vehicles in the Power Grid: Field Validation of Multiple Ancillary Services, IEEE Trans. Transp. Electrif. 3 (1) (2017) 201-209. doi :10.1109/TTE. 2016. 2616864 .

[27] O. Beaude, S. Lasaulce, M. Hennebel, I. Mohand-Kaci, Reducing the Impact of EV Charging Operations on the Distribution Network, IEEE Trans. Smart Grid 7 (6) (2016) 2666-2679. arXiv:1509.07056, doi:10.1109/TSG.2015.2489564.

[28] C. García Veloso, Real Time Voltage and Thermal Management of Low Voltage Distribution Networks through Plug-in Electric Vehicles, Master's thesis, Barcelona School of Industrial Engineering (2017).

[29] S. Sarabi, Contribution of Vehicle-to-Grid (V2G) to the energy management of the Electric Vehicles fleet on the distribution network, Ph.D. thesis, Génie électrique. Ecole nationale supérieure d'arts et métiers - ENSAM (nov 2016).

URL http://www . theses.fr/2016ENAM0050

[30] P. Richardson, D. Flynn, A. Keane, Local versus centralized charging strategies for electric vehicles in low voltage distribution systems, IEEE Trans. Smart Grid 3 (2) (2012) 1020-1028. arXiv:1008.2706, doi:10.1109/TSG.2012.2185523.

[31] J. Schlund, M. Pruckner, R. German, FlexAbility - Modeling and Maximizing the Bidirectional Flexibility Availability of Unidirectional Charging of Large Pools of Electric Vehicles, in: e-Energy 2020 - Proceedings of the 11th ACM International Conference on 
Future Energy Systems, Association for Computing Machinery, Inc, 2020, pp. 121-132. doi:10.1145/3396851.3397697.

[32] N. S. Pearre, H. Ribberink, Review of research on V2X technologies, strategies, and operations, Renew. Sustain. Energy Rev. 105 (2019) 61-70. doi:10.1016/j.rser. 2019.01 .047$.

[33] M. Petit, M. Hennebel, EV smart charging in collective residential buildings: The BienVEnu project, in: 2019 IEEE Milan PowerTech, PowerTech 2019, Institute of Electrical and Electronics Engineers Inc., 2019, pp. 1-6. doi:10.1109/PTC.2019. 8810464 .

[34] W. Jiang, Y. Zhen, A Real-Time EV Charging Scheduling for Parking Lots with PV System and Energy Store System, IEEE Access 7 (2019) 86184-86193. doi:10.1109/ ACCESS.2019.2925559.

[35] A. W. Thompson, Y. Perez, Vehicle-to-Everything (V2X) energy services, value streams, and regulatory policy implications, Energy Policy 137 (2019) 111136. doi: 10.1016/j.enpol.2019.111136.

[36] Europacable, An Introduction to Medium and Low Voltage Cables in Distribution Networks as support of Smart Grids, Tech. rep. (2014).

URL www . europacable.com

[37] C. Crozier, M. Deakin, T. Morstyn, M. McCulloch, Coordinated electric vehicle charging to reduce losses without network impedances, IET Smart Grid (may 2020). doi:10.1049/iet-stg.2019.0216.

[38] Enedis, ADEeF, Valorisation économique des Smart Grids Contribution des gestionnaires de réseau public de distribution 2017, Tech. rep., Enedis (2018).

[39] Energy Networks Association, Open Networks Project DSO Service Requirements: Definitions, Tech. rep. (2018).

URL https://www.nationalgrid.com/uk/electricity/balancing-services 
[40] S. M. Suhail Hussain, M. A. Aftab, I. Ali, T. S. Ustun, IEC 61850 based energy management system using plug-in electric vehicles and distributed generators during emergencies, Int. J. Electr. Power Energy Syst. 119 (2020) 105873. doi:10.1016/j. i jepes.2020.105873.

[41] University of Delaware. Communications and Public Affairs, Officials celebrate as electric vehicle-to-grid technology sells power to PJM power grid (2013) [Accessed: 201901-17].

URL http://www1.udel.edu/udaily/2013/apr/electric-vehicles-042613.html

[42] Parker Project, Parker Project Factsheet, Tech. rep. (2018).

URL WWW . parker-project.com

[43] Media Groupe PSA, GridMotion Project: reducing electric vehicle usage cost thanks to smart charging process, press release (2017) [Accessed: 2020-02-07].

URL https://media.groupe-psa.com/en/gridmotion-project-reducing-electric-vehicle-u

[44] O. Borne, Vehicle-To-Grid and Flexibility for Electricity Systems: from Technical Solutions to Design of Business Models, Ph.D. thesis, Université Paris-Saclay (2019).

[45] M. Wolinetz, J. Axsen, J. Peters, C. Crawford, Simulating the value of electric-vehiclegrid integration using a behaviourally realistic model, Nat. Energy 3 (2) (2018) 132139. doi:10.1038/s41560-017-0077-9.

[46] H. Lund, W. Kempton, Integration of renewable energy into the transport and electricity sectors through V2G, Energy Policy 36 (9) (2008) 3578-3587. arXiv:arXiv: 1011.1669v3, doi:10.1016/j.enpol.2008.06.007.

[47] A. Zecchino, K. Knezovic, M. Marinelli, Identification of conflicts between transmission and distribution system operators when acquiring ancillary services from electric vehicles, in: 2017 IEEE PES Innov. Smart Grid Technol. Conf. Eur. ISGT-Europe 2017 - Proc., Vol. 2018-Janua, IEEE, 2018, pp. 1-6. doi :10.1109/ISGTEurope.2017. 8260127. 
[48] E. Veldman, R. A. Verzijlbergh, Distribution grid impacts of smart electric vehicle charging from different perspectives, IEEE Trans. Smart Grid 6 (1) (2015) 333-342. doi:10.1109/TSG.2014.2355494.

[49] A. Bartolini, G. Comodi, D. Salvi, P. A. Østergaard, Renewables self-consumption potential in districts with high penetration of electric vehicles, Energy 213 (2020) 118653. doi:10.1016/j.energy.2020.118653.

[50] J. Dixon, W. Bukhsh, C. Edmunds, K. Bell, Scheduling electric vehicle charging to minimise carbon emissions and wind curtailment, Renew. Energy 161 (2020) 10721091. doi:10.1016/j.renene.2020.07.017.

[51] International Electrotechnical Commission, European Standard IEC 61851-1, Tech. rep. (2014).

[52] J. Dixon, K. Bell, Electric vehicles: Battery capacity, charger power, access to charging and the impacts on distribution networks, eTransportation 4 (2020) 100059. doi: $10.1016 / j$.etran.2020.100059.

[53] A. Thingvad, C. Ziras, M. Marinelli, Economic value of electric vehicle reserve provision in the Nordic countries under driving requirements and charger losses, J. Energy Storage 21 (2019) 826-834. doi:10.1016/j.est.2018.12.018.

[54] K. Knezović, M. Marinelli, Phase-wise enhanced voltage support from electric vehicles in a Danish low-voltage distribution grid, Electr. Power Syst. Res. 140 (2016) 274-283. doi:10.1016/j.epsr.2016.06.015.

[55] N. Leemput, F. Geth, J. Van Roy, J. Büscher, J. Driesen, Reactive power support in residential LV distribution grids through electric vehicle charging, Sustain. Energy, Grids Networks 3 (2015) 24-35. doi:10.1016/j.segan.2015.05.002.

[56] S. Gouraud, A. Szewczyk, V2G-AC-grid codes compliancy, from lab testing to field experiment, in: 32nd Electr. Veh. Symp., Renault, Lyon, 2019. 
[57] J. Kester, L. Noel, G. Zarazua de Rubens, B. K. Sovacool, Promoting Vehicle to Grid (V2G) in the Nordic region: Expert advice on policy mechanisms for accelerated diffusion, Energy Policy 116 (2018) 422-432. doi:10.1016/j.enpol.2018.02.024.

[58] A. W. Thompson, Economic implications of lithium ion battery degradation for Vehicle-to-Grid (V2X) services, J. Power Sources 396 (2018) 691-709. doi:10.1016/ j.jpowsour.2018.06.053.

[59] M. Dubarry, A. Devie, K. McKenzie, Durability and reliability of electric vehicle batteries under electric utility grid operations: Bidirectional charging impact analysis, J. Power Sources 358 (2017) 39-49. doi:10.1016/j.jpowsour.2017.05.015.

[60] D. Wang, J. Coignard, T. Zeng, C. Zhang, S. Saxena, Quantifying electric vehicle battery degradation from driving vs. vehicle-to-grid services, J. Power Sources 332 (2016) 193-203. doi:10.1016/j.jpowsour.2016.09.116.

[61] M. Jafari, A. Gauchia, S. Zhao, K. Zhang, L. Gauchia, Electric Vehicle Battery Cycle Aging Evaluation in Real-World Daily Driving and Vehicle-to-Grid Services, IEEE Trans. Transp. Electrif. 4 (1) (2018) 122-134. doi:10.1109/TTE.2017.2764320.

[62] K. Uddin, M. Dubarry, M. B. Glick, The viability of vehicle-to-grid operations from a battery technology and policy perspective, Energy Policy 113 (2018) 342-347. doi: 10.1016/J.ENPOL.2017.11.015.

[63] W. Cao, J. Wu, N. Jenkins, C. Wang, T. Green, Benefits analysis of Soft Open Points for electrical distribution network operation, Appl. Energy 165 (2016) 36-47. doi: $10.1016 / \mathrm{j}$. apenergy .2015.12.022.

[64] A. Procopiou, K. Petrou, Advanced Planning of PV-Rich Distribution Networks Deliverable 3: Traditional Solutions, Tech. rep., The University of Melbourne (2020).

[65] W. Kempton, J. Tomić, Vehicle-to-grid power implementation: From stabilizing the grid to supporting large-scale renewable energy, J. Power Sources 144 (1) (2005) 280294. doi:10.1016/j.jpowsour.2004.12.022. 
[66] L. Liu, F. Kong, X. Liu, Y. Peng, Q. Wang, A review on electric vehicles interacting with renewable energy in smart grid, Reneweable \& Sustainaible Energy Reviews 51 (2015) 648-661. doi:10.1016/j.rser.2015.06.036.

[67] J. K. Szinai, C. J. Sheppard, N. Abhyankar, A. R. Gopal, Reduced grid operating costs and renewable energy curtailment with electric vehicle charge management, Energy Policy 136 (2020) 111051. doi:10.1016/j .enpol.2019.111051.

[68] S. Moorman, T. Van 't Wel, T. Van Beek, The value of vehicle-to-grid (V2G) for distribution system congestion management, in: 32nd Electr. Veh. Symp., Lyon, 2019.

[69] G. Lacey, G. Putrus, A. Salim, The use of second life electric vehicle batteries for grid support, in: IEEE EuroCon 2013, IEEE, 2013, pp. 1255-1261. doi:10.1109/ EUROCON.2013.6625141.

[70] L. Canals Casals, M. Barbero, C. Corchero, Reused second life batteries for aggregated demand response services, J. Clean. Prod. 212 (2019) 99-108. doi:10.1016/j. jclepro.2018.12.005.

[71] M. Neaimeh, P. B. Andersen, Mind the gap- open communication protocols for vehicle grid integration, Energy Informatics 3 (1) (2020) 1. doi:10.1186/ s42162-020-0103-1.

[72] B. Christensen, M. Trahand, P. B. Andersen, O. J. Olesen, A. Thingvad, Integration of new technology in the ancillary service markets, Tech. rep., Technical University of Denmark, Department of Electrical Engineering (2018).

URL http://parker-project.com

[73] ElaadNL, EV Related Protocol Study v1.1, Tech. rep. (2017).

[74] Western Power Distribution, Electric Nation Customer Trial Final Report, Tech. rep. (2019).

[75] M. Zweistra, S. Janssen, F. Geerts, Large Scale Smart Charging of Electric Vehicles in Practice, Energies 13 (2) (2020) 298. doi:10.3390/en13020298. 
[76] CharIN e. V., Support of ISO/IEC 15118, Tech. rep. (2017).

URL www . charinev .org

[77] C. e.V., "The five levels of Grid Integration" - CharIN e.V. Grid Integration roadmap published (jan 2019).

[78] M. Knez, G. K. Zevnik, M. Obrecht, A review of available chargers for electric vehicles: United States of America, European Union, and Asia, Renew. Sustain. Energy Rev. 109 (2019) 284-293. doi:10.1016/j.rser.2019.04.013.

[79] Nissan International, Nissan, Enel and Nuvve Operate World's First Fully Commercial Vehicle-to-Grid Hub in Denmark, press release (aug 2016).

[80] CEER, Flexibility Use at Distribution Level A CEER Conclusions Paper, Tech. rep. (2018).

[81] A. Zecchino, M. Marinelli, Analytical assessment of voltage support via reactive power from new electric vehicles supply equipment in radial distribution grids with voltagedependent loads, Int. J. Electr. Power Energy Syst. 97 (2018) 17-27. doi:10.1016/ j.ijepes.2017.10.034.

[82] IEEE Standards Coordinating Committee 21, 1547-2018 - IEEE Standard for Interconnection and Interoperability of Distributed Energy Resources with Associated Electric Power Systems Interfaces (2018).

[83] INTERPLAN, Deliverable D2.2 Grid Code and regulation limitations, Tech. rep. (2019).

[84] S. Y. Hadush, L. Meeus, DSO-TSO cooperation issues and solutions for distribution grid congestion management, Energy Policy 120 (2018) 610-621. doi:10.1016/j. enpol.2018.05.065.

[85] UK Power Networks, Flexible distributed generation connections [Accessed: 2019-0208]. 
URL

https://www.ukpowernetworks.co.uk/electricity/

distribution-energy-resources/flexible-distributed-generation

[86] Enedis, Offres de raccordement intelligentes (2018) [Accessed: 2019-02-05]. URL https://www.enedis.fr/actualites/offres-de-raccordement-intelligentes

[87] R. Fonteijn, M. van Amstel, P. Nguyen, J. Morren, G. Maarten Bonnema, H. Slootweg, Evaluating flexibility values for congestion management in distribution networks within Dutch pilots, J. Eng. 2019 (18) (2019) 5158-5162. doi:10.1049/joe.2018.9314.

[88] Boletin Oficial del Estado, Disposición 1066 del BOE núm. 21 de 2020, Tech. rep. (2020).

URL https://www. boe.es

[89] Amsterdam Smart City, Flexpower Amsterdam (2018) [Accessed: 2019-01-10]. URL https://amsterdamsmartcity.com/projects/flexpower-amsterdam

[90] ACER, ACER Market Monitoring Report 2018 - Electricity and Gas Retail Markets Volume, Tech. rep., ACER (2019).

[91] I. Perez-Arriaga, C. Knittel, Utility of the Future, MIT Energy Initiative, Massachusetts Institute of Technology, Cambridge (MA), 2016.

URL energy . mit.edu/uof

[92] T. Schittekatte, Distribution network tariff design and active consumers : a regulatory impact analysis, Ph.D. thesis, Université Paris Saclay (2019).

URL https://tel.archives-ouvertes.fr/tel-02099785

[93] Q. Hoarau, Y. Perez, Network tariff design with prosumers and electromobility: Who wins, who loses?, Energy Econ. 83 (2019) 26-39. doi:10.1016/j. eneco.2019.05.009.

[94] E. Ramos Muñoz, G. Razeghi, L. Zhang, F. Jabbari, Electric vehicle charging algorithms for coordination of the grid and distribution transformer levels, Energy 113 (2016) 930-942. doi:10.1016/j . energy.2016.07.122. 
[95] R. A. Verzijlbergh, L. J. De Vries, Z. Lukszo, Renewable energy sources and responsive demand. Do we need congestion management in the distribution grid?, IEEE Trans. Power Syst. 29 (5) (2014) 2119-2128. doi:10.1109/TPWRS.2014.2300941.

[96] N. . Shao, S. . You, H. Segerberg, Integration of 100\% heat pumps and electric vehicles in the low voltage distribution network: A Danish case story, in: Proc. 3rd Int. Conf. Microgeneration Relat. Technol., Naples, 2013.

[97] Eurelectric, Dynamic pricing in electricity supply, Tech. rep. (2017).

[98] Octopus Energy, Agile Octopus [Accessed: 2020-02-05]. URL https://octopus .energy/agile/

[99] F. Salah, J. P. Ilg, C. M. Flath, H. Basse, C. van Dinther, Impact of electric vehicles on distribution substations: A Swiss case study, Appl. Energy 137 (2015) 88-96. doi: $10.1016 / \mathrm{j}$. apenergy .2014.09.091.

[100] A. Papavasiliou, Analysis of distribution locational marginal prices, IEEE Trans. Smart Grid 9 (5) (2018) 4872-4882. doi:10.1109/TSG. 2017.2673860.

[101] R. Mieth, Y. Dvorkin, Data-Driven Distributionally Robust Optimal Power Flow for Distribution Systems, IEEE Control Syst. Lett. 2 (3) (2018) 363-368. arXiv:1803. 04912, doi:10.1109/LCSYS.2018.2836870.

[102] S. Huang, Q. Wu, M. Shahidehpour, Z. Liu, Dynamic Power Tariff for Congestion Management in Distribution Networks, IEEE Transactions on Smart Grid 10 (2) (2018) 2148-2157. doi:10.1109/TSG.2018.2790638.

[103] M. Caramanis, E. Ntakou, W. W. Hogan, A. Chakrabortty, J. Schoene, Cooptimization of power and reserves in dynamic T\&D power markets with nondispatchable renewable generation and distributed energy resources, Proceedings of the IEEE 104 (4) (2016) 807-836. doi:10.1109/JPROC. 2016.2520758.

[104] CEER, CEER Paper on Electricity Distribution Tariffs Supporting the Energy Transition, Tech. rep. (2020). 
URL https://eur-lex.europa.eu/legal-content/EN/ALL/?uri=CELEX\% 3A32009R0714

[105] Enedis, TURPE 5 bis HTA/BT, Tech. rep. (2019).

[106] V. Van Lagen, Tariff setting methodology for electricity distribution in the Netherlands, Tech. rep., Authority for Consumers \& Markets, Vienna (oct 2017).

[107] Pwc, Smart Charging of electric vehicles : Institutional bottlenecks and possible solutions, Tech. rep., Pwc (2018).

[108] P. Cappers, J. Macdonald, J. Page, J. Potter, E. Stewart, E. O. Lawrence, Future Opportunities and Challenges with Using Demand Response as a Resource in Distribution System Operation and Planning Activities, Tech. rep., Lawrence Berkeley National Laboratory (2016).

[109] S. Haro, V. Aragonés, M. Martínez, E. Moreda, A. Morata, E. Arbós, J. Barquín, Toward Dynamic Network Tariffs: A Proposal for Spain, in: Innov. Disrupt. Grid's Edge How Distrib. Energy Resour. are Disrupting Util. Bus. Model, Elsevier Inc., 2017, pp. 221-239. doi:10.1016/B978-0-12-811758-3.00012-7.

[110] Consolidated Edison Company of New York, Orange and Rockland Utilities, Smart Home Rate REV Demonstration Project Implementation Plan, Tech. rep. (2018).

[111] UK Power Networks, Distribution Use of System charges (2020) [Accessed: 2021-02$04]$.

URL https://www.ukpowernetworks.co.uk/internet/en/about-us/duos/

[112] Netze BW GmbH, Preise für die Nutzung des Stromverteilnetzes der Netze BW GmbH, Tech. rep. (2020). URL https://www.netze-bw.de/unternehmen/veroffentlichungen\#3-1-1

[113] CWAPE, Tarifs de distribution (2020) [Accessed: 2021-02-04]. URL https://www.cwape.be/node/176\#grilles-tarifaires 
[114] Energy Networks Association, Flexibility in Great Britain [Accessed: 2020-03-26].

URL

https://www . energynetworks.org/electricity/futures/

flexibility-in-great-britain.html

[115] Enedis, Les flexibilités au service de la transition énergétique et de la performance du réseau de distribution, Tech. rep., Enedis (2019).

[116] REV Connect, Non-wires alternatives (2020) [Accessed: 2020-02-07].

URL https://nyrevconnect.com/non-wires-alternatives/

[117] Dashboard - Picloflex [Accessed: 2019-06-16].

URL https://www.picloflex.com/dashboard

[118] EPEX Spot, enera project: EWE and EPEX SPOT to create local market platform to relieve grid congestions, press release (feb 2018).

[119] GOPACS, Dutch grid operators launch GOPACS: a smart solution to reduce congestion in the electricity grid (jan 2019).

[120] S. O. Ottesen, P. Olivella-Rossell, P. Llloret, A. Hentunen, Invade Project D5.3 Simplified Battery operation and control algorithm, Tech. rep., INVADE (2017).

[121] Interflex Project, D7.1 D7.2 District architecture requirements, tested innovations and use case planning, Tech. rep. (2018).

[122] P. Olivella-Rosell, P. Lloret-Gallego, Í. Munné-Collado, R. Villafafila-Robles, A. Sumper, S. Ø. Ottessen, J. Rajasekharan, B. A. Bremdal, Local flexibility market design for aggregators providing multiple flexibility services at distribution network level, Energies 11 (4) (apr 2018). doi:10.3390/en11040822.

[123] F. Gonzalez Venegas, M. Petit, Y. Perez, Can DERs fully participate in emerging local flexibility tenders?, in: Int. Conf. Eur. Energy Mark. EEM, Vol. 2019-Septe, Ljubljana, 2019. doi:10.1109/EEM. 2019.8916343. 
[124] R. Fonteijn, P. Nguyen, J. Morren, J. Slootweg, Demonstrating a generic four-step approach for applying flexibility for congestion management in daily operation, Sustainable Energy, Grids and Networks (2020) 100378doi:10.1016/j.segan.2020.100378.

[125] F. Binot, D. Le, M. Petit, A Three-Phase Four-Wire State Estimator Algorithm for Low Voltage Networks Management, in: 25th Int. Conf. Electr. Distrib., Madrid, 2019, pp. 3-6. doi:10.34890/767.

[126] M. van Amstel, Flexibility system design for electric vehicles. Performing congestion management for the DSO, Ph.D. thesis, University of Twente (2018).

[127] S. P. Burger, J. D. Jenkins, C. Batlle, I. J. Pérez-Arriaga, Restructuring Revisited Part 1: Competition in Electricity Distribution Systems, Energy J. 40 (3) (jul 2019). doi:10.5547/01956574.40.3.sbur.

[128] J. A. Schachter, P. Mancarella, J. Moriarty, R. Shaw, Flexible investment under uncertainty in smart distribution networks with demand side response: Assessment framework and practical implementation, Energy Policy 97 (2016) 439-449. doi: 10.1016/j.enpol.2016.07.038.

[129] R. Moreno, B. Bezerra, H. Rudnick, C. Suazo-Martinez, M. Carvalho, A. Navarro, C. Silva, G. Strbac, Distribution Network Rate Making in Latin America: An Evolving Landscape, IEEE Power Energy Mag. 18 (3) (2020) 33-48. doi:10.1109/MPE. 2020. 2972667.

[130] E-Cube Strategy Consultants, Etude sur les perspectives stratégiques de l'énergie, Tech. rep. (2018).

URL http://fichiers.cre.fr/Etude-perspectives-strategiques/ 1SyntheseGenerale/Perspectives_Strategiques_du_secteur_de_l_energie_ Synthese_generale_FR.pdf

[131] CEER, Incentives Schemes for Regulating Distribution System Operators, including for innovation. A CEER Conclusions Paper, Tech. Rep. February (2018). 
URL https://www.ceer.eu/eer_consult/open_public_consultations/pc_on_ incentives_sche

[132] ACER, The Role of the DSO, Tech. Rep. May (2017).

[133] Ofgem, RIIO-ED1 network price control [Accessed: 2020-01-04]. URL https://www.ofgem.gov.uk/network-regulation-riio-model/ current-network-price-controls-riio-1/riio-ed1-network-price-control

[134] European Commission, Proposal for a Regulation of the European Parliament and the Council on the internal market for electricity, Tech. Rep. 5, Brussels (2016).

URL https://eur-lex.europa.eu/legal-content/EN/TXT/PDF/?uri=CELEX: 52016PC0861\{\&\}from=EN

[135] California Public Utilities Commission, California Smart Grid Annual Report to the Governor and the Legislature in Compliance with Public Utilities Code § 913.2, Tech. rep. (2019).

[136] NYSERDA, Reforming the energy vision (2014).

[137] Smart Electric Power Alliance, Peak Load Management Alliance, E4TheFuture, NonWires Alternatives Case Studies from Leading U.S. Projects, Tech. rep. (2018).

[138] S. P. Burger, J. D. Jenkins, C. Batlle, I. J. Perez-Arriaga, Restructuring Revisited Part 2: Coordination in Electricity Distribution Systems, Energy J. 40 (3) (jul 2019). doi:10.5547/01956574.40.3.jjen

[139] A. Satchwell, P. Cappers, L. Schwartz, E. Martin Fadrhonc, A Framework for Organizing Current and Future Electric Utility Regulatory and Business Models, Tech. rep., Ernest Orlando Lawrence Berkeley National Laboratory (2015).

[140] Commission de Régulation de l'Energie, Les réseaux électriques au service des véhicules électriques, Tech. rep. (2018). 
[141] SAE International, J3072: Interconnection Requirements for Onboard, UtilityInteractive Inverter Systems (2015). URL https://www . sae.org/standards/content/j3072_201505/

[142] Delaware Senate, SB 12 - An Act To Amend Title 26 Of The Delaware Code Relating To Generating Systems With The New Society Of Automotive Engineers Electric Safety Standard (2019) [Accessed: 2020-08-15].

URL https://openstates .org/de/bills/150/SB12/

[143] University of Delaware Energy Policy Team, UD Electric Vehicle R\&D Group's Public Comment on the NJ 2019 Draft Energy Master Plan (2019). URL https://nj.gov/emp/pdf/draft_emp/UniversityofDelawarecomments.pdf

[144] H. Gerard, E. I. Rivero Puente, D. Six, Coordination between transmission and distribution system operators in the electricity sector: A conceptual framework, Util. Policy 50 (2018) 40-48. doi:10.1016/j.jup.2017.09.011.

[145] M. B. Arias, S. Bae, Electric vehicle charging demand forecasting model based on big data technologies, Appl. Energy 183 (2016) 327-339. doi:10.1016/j.apenergy. 2016.08.080.

[146] P. Olivella-Rosell, R. Villafafila-Robles, A. Sumper, J. Bergas-Jané, Probabilistic Agent-Based Model of Electric Vehicle Charging Demand to Analyse the Impact on Distribution Networks, Energies 8 (5) (2015) 4160-4187. doi:10.3390/en8054160.

[147] J. Quirós-Tortós, L. F. Ochoa, B. Lees, A statistical analysis of EV charging behavior in the UK, in: 2015 IEEE PES Innov. Smart Grid Technol. Lat. Am. ISGT LATAM 2015, Institute of Electrical and Electronics Engineers Inc., 2016, pp. 445-449. doi: 10.1109/ISGT-LA.2015.7381196.

[148] C. Crozier, T. Morstyn, M. Deakin, M. McCulloch, The case for Bi-directional charging of electric vehicles in low voltage distribution networks, Appl. Energy 259 (feb 2020). doi:10.1016/j.apenergy.2019.114214. 
[149] M. Neaimeh, S. D. Salisbury, G. A. Hill, P. T. Blythe, D. R. Scoffield, J. E. Francfort, Analysing the usage and evidencing the importance of fast chargers for the adoption of battery electric vehicles, Energy Policy 108 (2017) 474-486. doi:10.1016/j.enpol. 2017.06 .033 .

[150] R. R. Desai, R. B. Chen, W. Armington, A pattern analysis of daily electric vehicle charging profiles: Operational efficiency and environmental impacts, J. Adv. Transp. (2018). doi:10.1155/2018/6930932.

[151] Y. E. Bouhassani, N. Refa, R. Van Den Hoed, Pinpointing the Smart Charging Potential for Electric Vehicles at Public Charging Points, in: 32nd Electr. Veh. Symp., Lyon, 2019.

[152] Z. J. Lee EE, T. Li, S. H. Low, Z. J. Lee, ACN-Data: Analysis and Applications of an Open EV Charging Dataset, in: Tenth Int. Conf. Futur. Energy Syst. (e-Energy '19), Phoenix, 2019. doi:10.1145/3307772.3328313.

[153] C. Morton, J. Anable, G. Yeboah, C. Cottrill, The spatial pattern of demand in the early market for electric vehicles: Evidence from the United Kingdom, J. Transp. Geogr. 72 (2018) 119-130. doi:10.1016/j.jtrangeo.2018.08.020.

[154] F. Heymann, V. Miranda, N. Neyestani, F. J. Soares, Mapping the Impact of Daytime and Overnight Electric Vehicle Charging on Distribution Grids, in: 2017 IEEE Veh. Power Propuls. Conf., IEEE, Belfort, 2017, pp. 1-6. doi:10.1109/VPPC.2017. 8330961 .

[155] F. Gonzalez Venegas, M. Petit, Y. Perez, Impact of Non-Systematic Electric Vehicle Charging Behaviour on a Distribution Substation, in: Proc. 2019 IEEE PES Innov. Smart Grid Technol. Eur. ISGT-Europe 2019, Bucharest, 2019. doi:10.1109/ ISGTEurope. 2019.8905710.

[156] T. Gnann, A. L. Klingler, M. Kühnbach, The load shift potential of plug-in electric vehicles with different amounts of charging infrastructure, J. Power Sources 390 (2018) 20-29. doi:10.1016/j.jpowsour.2018.04.029. 
[157] F. Gonzalez Venegas, M. Petit, Y. Perez, Quantifying the participation of EV fleets in local flexibility tenders, in: Proc. CIRED 2020 Berlin Work., Berlin, 2020.

[158] J. Bailey, J. Axsen, Anticipating PEV buyers' acceptance of utility controlled charging, Transp. Res. Part A Policy Pract. 82 (2015) 29-46. doi:10.1016/j.tra.2015.09.004

[159] C. Will, A. Schuller, Understanding user acceptance factors of electric vehicle smart charging, Transp. Res. Part C Emerg. Technol. 71 (2016) 198-214. doi:10.1016/j. $\operatorname{trc} .2016 .07 .006$.

[160] EMPOWER H2020, Deliverable 2.3 Models of prosumer acceptance, Tech. rep. (2017).

[161] FleetCarma, SmartCharge Platform - How the Program Works (2020) [Accessed: 2020-07-20].

URL https://www.fleetcarma. com/smartcharge/

[162] Ofgem, Implications of the Transition to Electric Vehicles, Tech. rep. (2018). arXiv:yd, doi:10.1016/S0022-3182(80)80003-3.

URL https : //wWw . of gem.gov . uk/of gem-publications/136142

[163] GOV.UK, £30 million investment in revolutionary V2G technologies - GOV.UK (2018) [Accessed: 2018-12-14].

URL https : //www .gov .uk/government/news/30-million-investment-in-revolutionary-v2g-

[164] A. Alyousef, D. Danner, F. Kupzog, H. de Meer, Enhancing power quality in electrical distribution systems using a smart charging architecture, Energy Informatics 1 (S1) (2018) 28. doi:10.1186/s42162-018-0027-1. 